\title{
JOINT USER GROUPING AND TIME ALLOCATION FOR NOMA WITH WIRELESS POWER TRANSFER
}

by

\section{Mehak Basharat}

BS Telecom Engineering, National University of Computer and Emerging Sciences, Islamabad, Pakistan, 2009

MS Computer Engineering, University of Engineering and Technology, Taxila, Pakistan, 2012

\author{
A thesis \\ presented to Ryerson University \\ in partial fulfillment of the \\ requirements for the degree of \\ Master of Applied Science \\ in the Program of
}

Electrical and Computer Engineering

Toronto, Ontario, Canada

(C)Mehak Basharat 2017 


\section{AUTHOR'S DECLARATION FOR ELECTRONIC SUBMISSION OF A THESIS}

I hereby declare that I am the sole author of this thesis. This is a true copy of the thesis, including any required final revisions, as accepted by my examiners.

I authorize Ryerson University to lend this thesis to other institutions or individuals for the purpose of scholarly research.

I further authorize Ryerson University to reproduce this thesis by photocopying or by other means, in total or in part, at the request of other institutions or individuals for the purpose of scholarly research.

I understand that my thesis may be made electronically available to the public. 


\begin{abstract}
Joint User Grouping and Time Allocation for NOMA with Wireless Power Transfer
\end{abstract}

Master of Applied Science

2017

Mehak Basharat

Electrical and Computer Engineering

Ryerson University

Non-Orthogonal Multiple Access (NOMA) has recently been explored to address the challenges in 5G networks such as spectral efficiency, large number of devices, etc. Further, energy harvesting is a promising solution to address the challenges for energy efficiency in $5 \mathrm{G}$ networks. In this thesis, joint user grouping, power allocation, and time allocation for NOMA with RF energy harvesting is investigated. We mathematically modeled a framework to optimize user grouping, power allocation, and time allocation for energy harvesting and information transfers. The objective is to maximize data rate while satisfying the constraints on minimum data rate requirement of each user and transmit power. We adopted mesh adaptive direct search (MADS) algorithm to solve the formulated problem. The user grouping in MADS is comparable with the exhaustive search which is computationally very complex. The thesis is supported with simulation results in terms of user grouping, power allocation, user rate, and time sharing. 


\section{Acknowledgements}

I would like to express my sincere gratitude to my supervisor, Prof. Alagan Anpalagan for his continuous encouragement and support. He was always generous with his invaluable time and always motivating me. It was a great privilege to work with him. I would like to acknowledge the Department of Electrical and Computer Engineering and the School of Graduate Studies at Ryerson University for their support in terms of financial aid, and work experience as a graduate assistant. I would also like to thank my defense committee for taking the time and effort to review my work and provide me with their insightful comments. I am also thankful to my colleagues in the WAN group, specially Dr. Muhammad Naeem, I am lucky to be a part of this group where a team spirit truly prevails.

I can never find the words to thank my husband, Waleed Ejaz, who was amazingly patient and understanding and who helped me through the most difficult times of my life. He also offered me his advice and his valuable help whenever I needed it. My special thanks goes to my parents. My mother who is always a great source of love and motivation and my father who not only believed in me but was always supportive. I can never thank them enough for everything that they did for me. I would also like to take this opportunity to thank my siblings for their love and encouragement. Thank you for all the little and great things you have done for me. Last but not the least, I would like to appreciate my son here, Aahil, who is a constant source of joy and who always gives me a reason to smile. 


\section{Table of Contents}

Author's Declaration ................... i ii

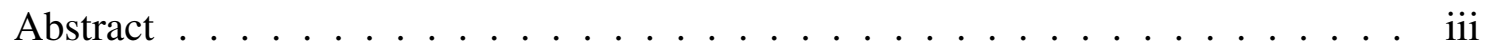

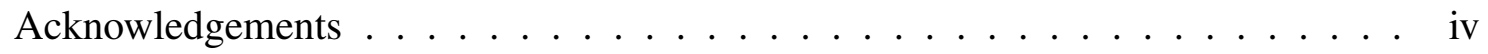

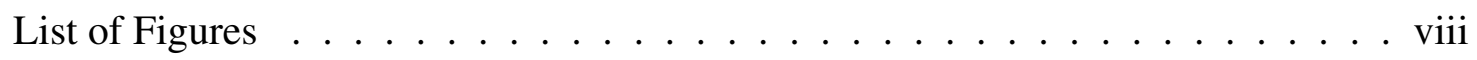

List of Tables ........................ ix

1 Introduction $\quad 1$

1.1 Non Orthogonal Multiple Access (NOMA) . . . . . . . . . . . . . . . 2

1.1 .1 NOMA in $5 \mathrm{G} \ldots \ldots \ldots \ldots$

1.1.2 NOMA with Energy Harvesting . . . . . . . . . . . . . . 5

1.2 Thesis Contributions $\ldots \ldots \ldots \ldots$

1.3 Thesis Organization . . . . . . . . . . . . . . . . 7

2 Literature Review of NOMA with User Grouping and NOMA with Energy Harvest$\begin{array}{ll}\text { ing } & 8\end{array}$

2.1 Taxonomy of Multiple Access Schemes . . . . . . . . . . . . . . . . 9

2.1.1 Orthogonal Multiple Access . . . . . . . . . . . . . . . . 10

2.1.2 Non-Orthogonal Multiple Access . . . . . . . . . . . . . . . 11

2.2 User Grouping and Power allocation in NOMA . . . . . . . . . . . . . . . 15

2.3 Energy Harvesting and Wireless Power Transfer in NOMA . . . . . . . . . . . . 18

3 System Model and Problem Formulation $\quad 20$ 
3.1 Network Model . . . . . . . . . . . . . . . . . . . . . . . . . 20

3.2 Problem Formulation $\ldots \ldots \ldots \ldots \ldots$

4 Proposed Scheme and Comparison 2

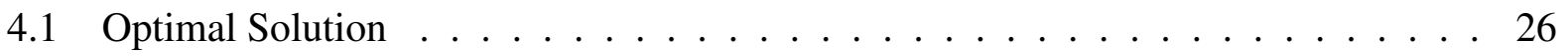

$4.1 .1 \quad$ User Grouping . . . . . . . . . . . . . . . . . . . . . . . 26

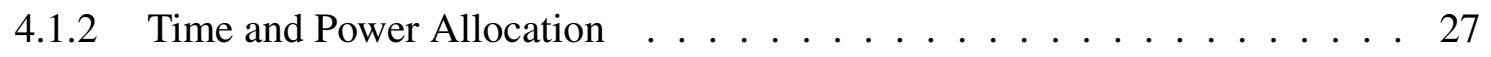

4.1.3 Mesh Adaptive Direct Search Algorithm . . . . . . . . . . . . . . . . . . 28

4.2 Performance Analysis . . . . . . . . . . . . . . . . . . . . . . 31

4.2.1 Comparison between MADS and ESA algorithm . . . . . . . . . 31

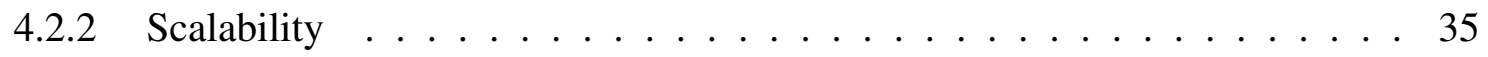

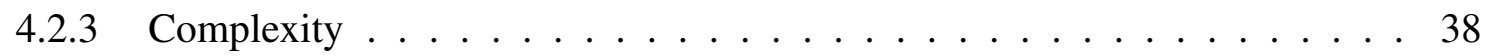

5 Conclusions and Future work $\quad 39$

5.1 Conclusions . . . . . . . . . . . . . . . . . . . . . . 39

5.2 Future Research Directions . . . . . . . . . . . . . . . . . . . . 40

5.2.1 Optimal/ Low Complexity Receiver Design . . . . . . . . . . . . . . . 40

5.2 .2 NOMA with imperfect CSI . . . . . . . . . . . . . . . . . . 40

5.2.3 MIMO-NOMA and Internet of Things . . . . . . . . . . . . . . 41

5.2.4 Optimal Wireless Power Transfer . . . . . . . . . . . . . . . . . . 41

$\begin{array}{ll}\text { Appendix } & 43\end{array}$

$\begin{array}{lr}\text { Bibliography } & 44\end{array}$ 


\section{List of Figures}

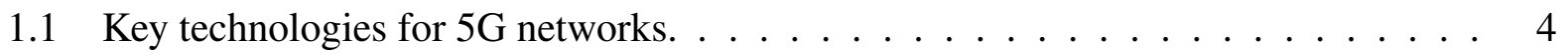

2.1 Taxonomy of multiple access schemes. . . . . . . . . . . . . . . . . 10

3.1 An illustration of network that consists of $K$ users and $N$ resource blocks for NOMA with wireless power transfer. . . . . . . . . . . . . . . . 22

4.1 Performance comparison of MADS algorithm versus ESA for $\mathrm{K}=6, \mathrm{~N}=2$, and $R^{\text {min }}=125 \mathrm{kbps}$ : (a) resource block index versus user index, (b) user power for each resource block, (c) user rate for each user, and (d) time sharing for each resource block. . . . . . . . . . . . . . . . . . . . 32

4.2 Performance comparison of MADS algorithm versus ESA for $\mathrm{K}=6, \mathrm{~N}=2$, and $R^{\text {min }}=1$ Mbps: (a) resource block index versus user index, (b) user power for each resource block, (c) user rate for each user, and (d) time sharing for each resource

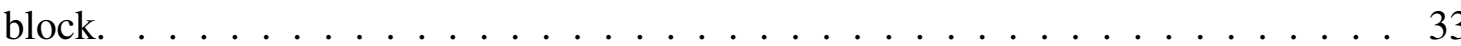

4.3 Performance comparison of MADS algorithm versus ESA for $\mathrm{K}=5, \mathrm{~N}=3$, and $R^{\text {min }}=125 \mathrm{kbps}$ : (a) resource block index versus user index, (b) user power for each resource block, (c) user rate for each user, and (d) time sharing for each resource block. . . . . . . . . . . . . . . . . . . . . . 34 
4.4 Performance comparison of MADS algorithm versus ESA for $K=5, N=3$, and $R^{\text {min }}=2 \mathrm{Mbps}$ : (a) resource block index versus user index, (b) user power for each resource block, (c) user rate for each user, and (d) time sharing for each resource

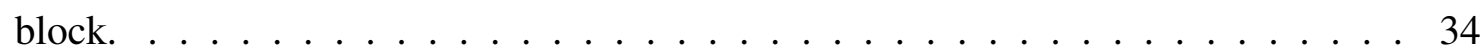

4.5 Performance of MADS algorithm for $\mathrm{K}=24, \mathrm{~N}=2$, and $R^{\min }=125 \mathrm{kbps}$ : (a) resource block index versus user index, (b) user power for each resource block, (c) user rate for each user, and (d) time sharing for each resource block. . . . . . . . . . . . 36

4.6 Performance of MADS algorithm for $\mathrm{K}=24, \mathrm{~N}=1$, and $R^{\text {min }}=1 \mathrm{Mbps}$ : (a) resource block index versus user index, (b) user power for each resource block, (c) user rate for each user, and (d) time sharing for each resource block. . . . . . . . . . . . . 37

4.7 Performance comparison of MADS algorithm versus ESA for $K=24, N=2$, and $R^{\min }=1 \mathrm{Mbps}$ : (a) resource block index versus user index, (b) user power for each resource block, (c) user rate for each user, and (d) time sharing for each resource

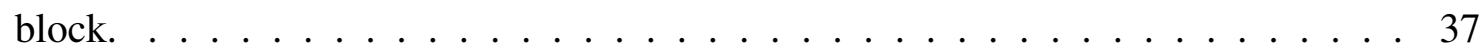




\section{List of Tables}

2.1 Existing Surveys and Comparisons on NOMA. . . . . . . . . . . . . 9

3.1 Description of the symbols used in the model. . . . . . . . . . . . . . . 23

4.1 Simulation parameters. . . . . . . . . . . . . . . 32

4.2 Complexity comparison of ESA versus MADS algorithm. . . . . . . . . . . 38 


\section{List of Abbreviations}

\begin{tabular}{|c|c|}
\hline Acronyms & Description \\
\hline $\mathrm{BNC}$ & Binary network coding \\
\hline $\mathrm{BS}$ & Base station \\
\hline $\mathrm{CR}$ & Cognitive radio \\
\hline CSI & Channel state information \\
\hline FDMA & Frequency division multiple access \\
\hline KPI & Key Performance Indicators \\
\hline LDPC & Low-density parity check \\
\hline LDS & Low-density spreading \\
\hline MAMRC & Multiple-access multiple relay channel \\
\hline MAP & Maximum a posteriori \\
\hline MC-LDSMA & Multi-carrier low-density spreading multiple access \\
\hline MILP & Mixed integer linear programming \\
\hline MIMO & Multiple-input multiple-output \\
\hline MPDA & Message passing decoding algorithm \\
\hline MUSA & Multi-user shared access \\
\hline OFDMA & Orthogonal frequency division multiple access \\
\hline OMA & Orthogonal multiple access \\
\hline PDMA & Pattern division multiple access \\
\hline PEP & Pair-wise Error Probability \\
\hline SCFDMA & Single-carrier FDMA \\
\hline SCMA & Sparse code multiple access \\
\hline $\mathrm{SIC}$ & Successive interference cancellation \\
\hline SISO & Single input single-output \\
\hline STBC & Space-time block code \\
\hline SWIPT & Simultaneous wireless information and power transfer \\
\hline
\end{tabular}




\section{Chapter 1}

\section{Introduction}

Multiple access schemes play an important role to distinguish various wireless systems from generation to generation. The frequency-division multiple access (FDMA) is a predominant orthogonal multiple access (OMA) scheme for the first generation of cellular networks. The second generation systems are mainly based on time-division multiple access (TDMA). Similarly, the third generation cellular networks operated on the code-division multiple access (CDMA) and, orthogonal frequency-division multiple access (OFDMA) is the ground for the fourth generation systems. Users are assigned orthogonal resources to minimize the inter-user interference in the traditional multiple access schemes. This can be done in any domain (code, time or frequency domain). OFDM has been widely explored and applied in various wireless communication systems to meet their requirements. In OFDM, the resources are divided into orthogonal sub-channels and then assigned to each user. Although OFDM is successfully being utilized in practical communication systems including LTE, yet with the rapid advancement in the technology, have changed the requirements of wireless communication all together [1,2]. One of the drawbacks of OMA schemes that it can support the maximum number of users limited by the number of resources and their scheduling. Mobile Internet has grown very rapidly and it is anticipated to increase even faster by 2020. Therefore, it is very crucial to efficiently utilize the resources in order to meet the requirements of this traffic explosion. 
Upon the conclusion of the fourth generation (4G) cellular network standardization tasks in 2011, research direction has started to shift systematically towards forthcoming fifth generation (5G) communication networks [3]. Just recently, research done in this area have been predominantly concerned with devising state-of-the-art, innovative, as well as disruptive concepts and technologies, pursuing leaps and strides beyond that of present-day 4G systems and their known limitations. The requirements of 5G wireless communication system include spectral efficiency, energy efficiency, low latency, tremendous connectivity, etc. In order to fulfill the demands of 5G, non-orthogonal multiple access (NOMA) is being considered [4]. NOMA offers enormous con-

nectivity and spectral efficiency; however, receiver complexity is slightly increased. Various users can share resources (time and frequency) in NOMA using power or code domain multiplexing.

\subsection{Non Orthogonal Multiple Access (NOMA)}

In NOMA, the data that is transmitted on the channel is actually the sum of information of all the users [5]. This collective data of all the users is also termed as superposition coded signal which is transmitted from the base station. The effective channel gain of each user determines their position in the queue. For example, the user with the highest effective channel gain gets the priority in the sequence and the user with the lowest gets the least. Rest of the users are scheduled in the same fashion between them. The benefit of NOMA is that it facilitates the user with the weak signal by assigning it a higher fraction of power $[4,6]$. In the conventional system where OMA schemes are used, users cannot access a particular frame once it has been reserved for a particular user. This adversely affects the overall throughput of the system. On the contrary, while implementing NOMA the user with stronger signal can transmit data on a pre-occupied slot already assigned to a user with weaker signal [7]. The performance of the weaker user will not be seriously affected as it will be undergoing the effect of channel fading anyway. The user with stronger signal avoids the interference caused by the poor signal with the application of successive interference cancellation (SIC) operation [8]. Hence, the overall data rate of the system can be improved with efficient 
resource allocation.

Several features of NOMA distinguish it from conventional OMA schemes:

- Spectral Efficiency: One of the key features of NOMA is spectral efficiency in which users share the frequency-time and are also multiplexed in the power domain, and thus increases spectral efficiency. Depending on the channel gain, the power is allocated to users for transmission. Analysis of uplink spectral efficiency for NOMA is presented in [9]. A closedform expression for spectral efficiency is proposed according to the accurate Gaussian-based evaluation. Likewise, the analysis for downlink spectral efficiency for NOMA is presented in [10] and a solution is proposed that leads to the spectral efficiency within finite number of operations for NOMA.

- Massive Connectivity: There is no constraint on the number of users that can be supported in NOMA. The resources in NOMA have the tendency of supporting multiple users. Therefore, NOMA can support massive connectivity when compared with OMA. For example, sparse code multiple access (SCMA) is proposed as a unique and versatile constellation design which can be overloaded to support massive connectivity [5]. Similarly, the performance of multi-user shared access (MUSA) is good enough keeping the overloading factor up to $300 \%[11]$.

- Low Latency and Signaling Cost: The typical OMA system is based on allowing transmission when a user sends a request for scheduling to the BS and then performs scheduling optimization for uplink transmission before granting the resources over the downlink channel. This will result in more delayed signal transmission and signaling cost [7]. On the contrary, when dealing with the up-link strategies in NOMA, dynamic scheduling like OMA is not required in some schemes. In the case of NOMA, the grant-free uplink transmission can significantly reduce latency and signaling overhead. Furthermore, NOMA can support users with different channel state information (CSI) in a timely manner which results in low latency [5]. 

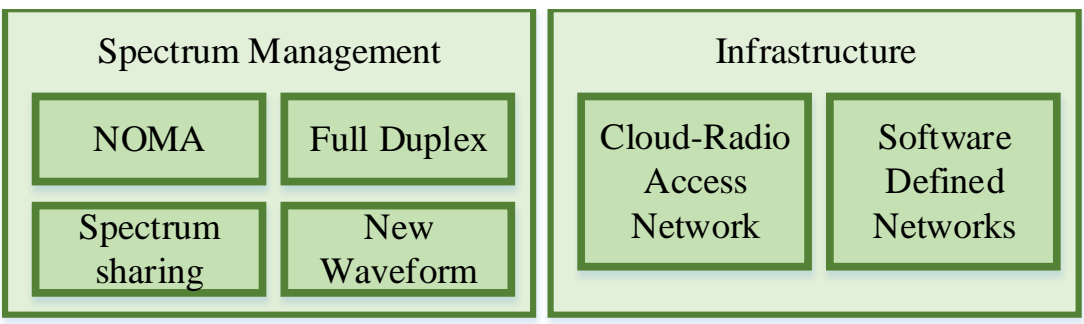

Flexible and Efficient Physical Layer

Figure 1.1: Key technologies for $5 \mathrm{G}$ networks.

We can classify NOMA in three types: power domain, constellation domain, and code domain. In power domain NOMA, the modulated symbols of different users are superposed with different transmit power simultaneously using the same frequency and spatial resource [12-15]. In constellation domain NOMA, multiple users can be multiplexed according to appropriately designed patterns and then map them to higher level constellation [16]. SCMA [17], low-density spreading (LDS) with multiple access and code domain multiplexing are discussed in [18, 19], MUSA [7]. Authors in [20] have done a detailed study on some additional multiple access schemes e.g. pattern division multiple access (PDMA) and bit division multiplexing (BDM).

\subsubsection{NOMA in 5G}

Key technologies for 5G networks are depicted in Fig. 1.1. Recently, various NOMA schemes have been designed to facilitate the future demand of data traffic. NOMA for single-input-single-output (SISO) systems is explored by many researchers. In [21], NOMA is investigated for downlink of a cellular system. The authors have derived equations for the average data rate and outage probability for every user assuming the power is fixed. A user may be in outage only if the targeted data rate is not chosen intelligently. Lan et al. [22] has compared the effect of user's velocity and error propagation for multiple-input-multiple-output (MIMO) systems incorporating NOMA. Their outcomes clearly illustrate that during error propagation for not very favorable situations, NOMA performs better than conventional OMA. NOMA for the downlink was studied in Chen 
et al. [23]. The study was done assuming that both the BS and receivers have two antennas. Precoding matrices which are conventionally used for minimum-mean-squared-error (MMSE) do not guarantee to achieve efficient data rate for a given order of user. Timotheou et al. [24] considered the problem from the fairness perspective and worked on the power allocation for SISO system in NOMA. Ding et al. on the other hand, researched MIMO-NOMA in [5]. Mathematical derivations were also shown for outage expectation for fixed and optimal power allocation. In [25], work has been done to execute NOMA keeping the functionality of OFDMA at the back end. It has been concluded in [26], that NOMA in 5G can achieve increased spectral efficiency. However, this will also result in a higher complexity of the receiver design.

\subsubsection{NOMA with Energy Harvesting}

Energy harvesting is considered as a promising solution to address the energy issues in 5G networks. The idea of energy harvesting is to gather energy from sources present in the surrounding such as RF signals, solar, wind, etc. In RF energy harvesting, users can harvest from ambient RF sources. The amount of energy harvested by users depends on the transmit power of RF sources, harvesting circuit, and propagation properties of the environment [27]. Harvesting circuit converts RF energy to DC which results in some loss. Thus, harvesting efficiency depends on the harvesting circuit and power consumed in reception by user. Time allocation for information transfer along with energy harvesting has attracted attention of many researchers [28]. For instance, authors in [29] have implemented a energy harvesting relay which is based on time switching and power splitting relaying. In [30] authors investigated time allocation methods with NOMA in up-

link communication system to improve the throughput of all users. Another approach proposed by authors in [31] is to prepare energy harvesting relays from NOMA users located near the base stations in order to help the users that are at a distance.

There are certain challenges associated with the NOMA in 5G including dynamic user grouping, power allocation and time allocation for energy harvesting. In NOMA, multiple users can use the same resource simultaneously which may result in co-channel interference. Therefore, 
the users can be divided into groups in NOMA based on several conditions such as co-channel interference, data rate requirement, etc. The user grouping along with power allocation when done efficiently can reduce the effect of interference and increase spectral efficiency [32]. However, 5G also constitutes energy efficiency challenges, because of the increase in number of wireless devices. Therefore, it is important to investigate dynamic user grouping, power allocation and time allocation for NOMA in 5G for energy harvesting.

In NOMA with RF energy harvesting, joint user grouping and time allocation (for information transfer and energy harvesting) is the indispensable design issue. Until now, researchers investigated either user grouping [32-38] or time allocation for information transfer and energy harvesting [30,31,39-42]. Therefore, it is important to investigate joint user grouping and time allocation for NOMA with RF energy harvesting. Thus, this thesis focuses on developing a joint user grouping and time allocation scheme for NOMA systems with RF energy harvesting. Until now, researchers investigated either user grouping or time allocation for information transfer and energy harvesting, in NOMA systems.

\subsection{Thesis Contributions}

The key contributions of this thesis can be summarized in the following points:

- For NOMA with RF energy harvesting, we mathematically model a framework for NOMA having energy harvesting capability. The mathematical modeling includes grouping of users in their respective resource blocks, and an optimized power and time allocation to achieve maximum system throughput. This model ensures minimum throughput of each admitted user and user power in each resource block.

- The proposed mathematical framework belongs to a type of optimization problem, called mixed integer non-linear programming (MINLP) and this type of problems are generally NP-hard. Thus, to get optimal solution we need to enumerate all possible assignments of users in their respective resource blocks which is computationally very expensive. Moreover, 
computational complexity increases exponentially with the number of users and resource blocks. We adopted mesh adaptive direct search (MADS) algorithm which provide epsilon optimal solution.

- Finally, we evaluate the performance of joint user grouping and time allocation for NOMA with RF energy harvesting using MADS algorithm. Simulation results using MADS algorithm are compared with the optimal solution obtained by exhaustive search method.

\subsection{Thesis Organization}

The rest of the thesis is organized as follows. We briefly review related works in Chapter 2. Chapter 3 introduces the system model. Then, we present strategies for joint user grouping and time sharing in NOMA with wireless power transfer in Chapter 4 . We illustrate the simulation results and discuss performance evaluation in Chapter 5. Finally, Chapter 6 states the conclusions. 


\section{Chapter 2}

\section{Literature Review of NOMA with User}

\section{Grouping and NOMA with Energy}

\section{Harvesting}

It is evident from the introduction that research in NOMA has a broader scope. NOMA schemes for $5 \mathrm{G}$ have been studied in recent years, however, research is scattered and usually focused on a narrow set of issues $[7,8,19,21,24,43-45]$. A comprehensive survey of power-domain NOMA in 5G systems is presented in [4]. Authors also discussed the implementation issues and performance of NOMA when integrated with existing wireless communication techniques such as cooperative communications, MIMO, network coding, etc. In [7], authors discussed and presented a detailed comparison for existing NOMA solutions for $5 \mathrm{G}$ networks. In addition, multiple directions for future study and challenges in NOMA such as the efficient spreading sequences, receiver design, etc. have been stated. In [25], promising NOMA schemes and non-orthogonal waveform modulation techniques for $5 \mathrm{G}$ mobile communications are studied and their future development is analyzed. In [26], authors have compared the three NOMA schemes (SCMA, MUSA, and PDMA) keeping in mind the up-link BER. It is concluded that SCMA outperforms its counterparts due to the near-optimal design of sparse codewords and near-optimal message passing algorithm receiver. 
Table 2.1: Existing Surveys and Comparisons on NOMA.

\begin{tabular}{ccl}
\hline Ref. & Year & Remarks \\
\hline$[4]$ & 2016 & $\begin{array}{l}\text { A comprehensive survey of power-domain NOMA in 5G systems is } \\
\text { presented. } \\
\text { A comprehensive comparison of existing NOMA solutions is provided. } \\
\text { In addition, future research trends and challenges for NOMA are high- } \\
\text { lighted. }\end{array}$ \\
[25] 2015 & 2015 & $\begin{array}{l}\text { Promising NOMA schemes and non-orthogonal waveform modulation } \\
\text { techniques for 5G mobile communications are studied and their future } \\
\text { development is analyzed. } \\
\text { A study of NOMA schemes is performed. The results are compared } \\
\text { subject to uplink bit error rate. } \\
\text { A comprehensive comparison for three interference cancellation } \\
\text { schemes (Conventional (SIC) scheme, soft-in soft-out parallel interfer- } \\
\text { ence cancellation (SISO-PIC), and a hybrid interference cancellation } \\
\text { (HIC) for NOMA is presented. }\end{array}$ \\
[46] 2016$]$ & 2015
\end{tabular}

On the other hand, the performance of SCMA and PDMA is similar. In [46], authors presented a comprehensive comparison for three interference cancellation schemes: 1) conventional SIC scheme, 2) soft-in-soft-out parallel interference cancellation (SISO-PIC), and 3) a hybrid interference cancellation (HIC) for NOMA. The performance of these schemes is compared in terms of error propagation, detection delay, and complexity. It is concluded that HIC is a trade-off scheme for the given performance parameters.

It is worth mentioning here that this survey is exclusive to optimization related issues for NOMA in 5G networks, whereas existing surveys and comparisons $[4,7,25,26,46]$ are broad based and discuss many aspects of NOMA in 5G networks. Therefore, the most pertinent surveys have been selected and reviewed in comparison to ours. A list of existing surveys and comparison studies is provided in Table 2.1.

\subsection{Taxonomy of Multiple Access Schemes}

Taxonomy of multiple access schemes is described in Fig. 2.1. There are two main categories of multiple access schemes named as 1) orthogonal and 2) non-orthogonal multiple access schemes. 


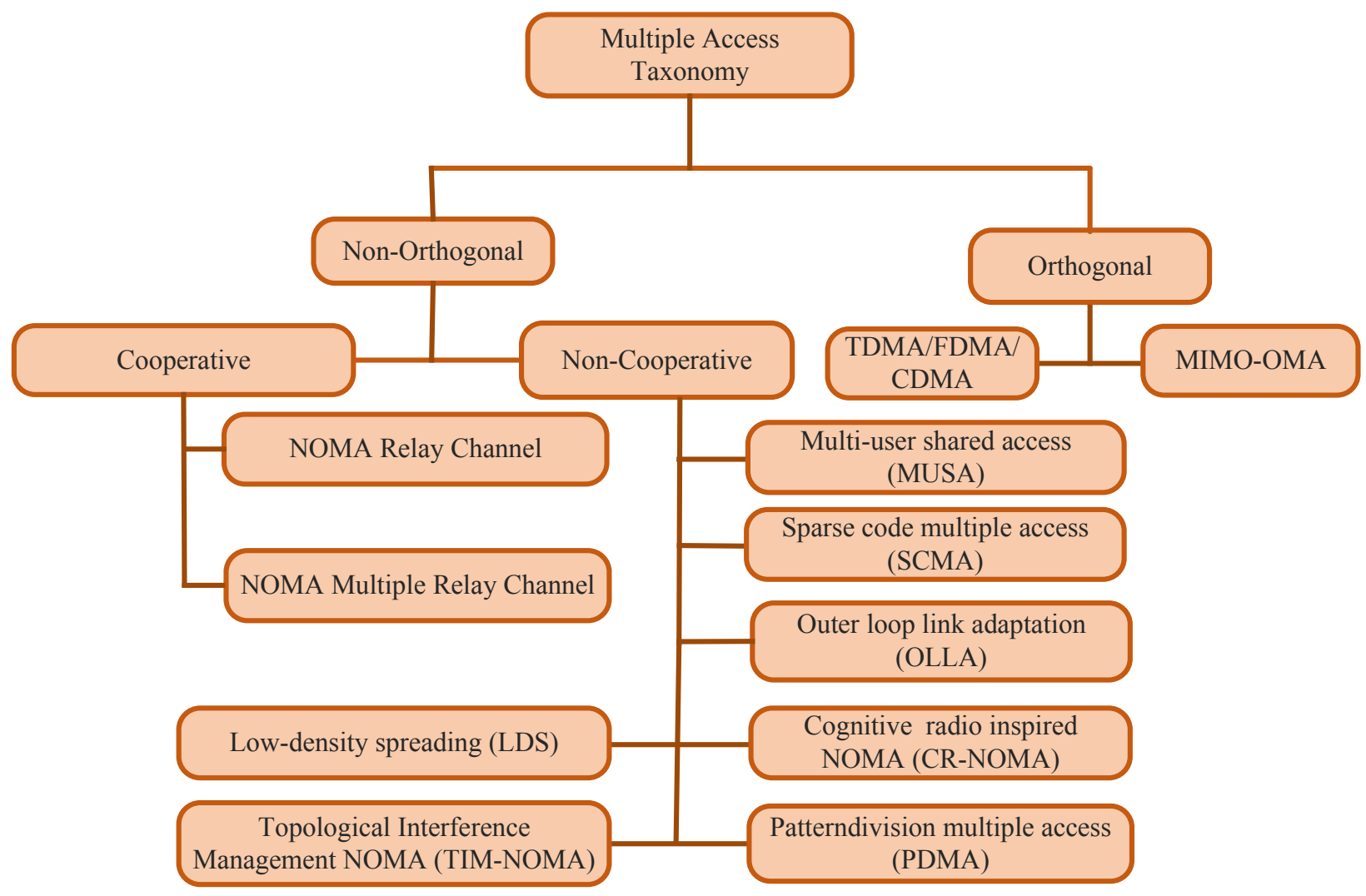

Figure 2.1: Taxonomy of multiple access schemes.

In this section, we will describe different types of multiple access schemes in each category.

\subsubsection{Orthogonal Multiple Access}

Orthogonal frequency division multiple access (OFDMA) is a type of OFDM scheme which is used for multiple users. Sub-carriers are allocated to each user in order to achieve multiple access in OFDMA. Adaptive assignment of a user-to-sub carrier can be accomplished based on the feedback received about the channel condition [47]. This assignment if done rapidly can further improve the performance of OFDM robustness to narrow band co-channel interference and fast fading. OFDMA offers several advantages for the wireless network such as scalability, use of multiple antennas, etc.

In time division multiple access (TDMA), users of the same network can share the same chan- 
nel. However, multiple time slots are used to transmit signal. The users forward their data simultaneously using their own respective time slot. A combination of time domain and frequency domain is also outlined as OFDMA. The division of resources can be done in both frequency and time domains [48]. In code-division multiple access (CDMA), multiple users can transmit their data simultaneously on the same channel [49]. CDMA employs spread-spectrum technology with a special coding scheme where every user is assigned a code. This is done to achieve minimum interference. On the other hand, MIMO techniques can enhance the spectral efficiency of OMA by using multiple transmit and receive antennas [50].

\subsubsection{Non-Orthogonal Multiple Access}

The underlying concept of NOMA is very divergent as compared to the traditionally studied OMA schemes. NOMA supports numerous users to communicate with the same code and frequency simultaneously with varying power levels. As stated earlier in NOMA, power allocation is according to the channel conditions. This means that users which are experiencing better channel gains will be assigned less power. Every user, however, will extract its own data with the application of SIC. NOMA is further classified into cooperative and non-cooperative schemes.

\section{Cooperative NOMA}

In cooperative NOMA, the prior information available in NOMA systems is fully exploited. In [51], authors concluded that the cooperative NOMA relay scheme performs much better under the presence of slow fading source-to-relay link as compared to the non-cooperative method. In [52], authors supposed that the BS's cooperate with each other for the down link transmission. Furthermore, for cooperative NOMA system interference among the cell-edge users is also investigated. In [53], authors aimed at enhancing the system capacity while using a combination of cooperative NOMA and dirty paper coding (DPC) for the resource scheduling optimization problem. Gen-

erally, cooperative NOMA can operate in two modes, NOMA relay channel, and multiple relay channel. 
NOMA Relay Channel: Studies have shown that relay implementation in a wireless network has worked effectively to cater the effect of shadowing and fading on the transmitted data. A transmission scheme in cooperative NOMA is proposed in [45]. The users with good channel conditions are considered as relays to reinforce the efficiency of data transmission for the users with weak channel conditions. Results are elaborated using successive detection strategy at the receiver side which indicates that the users with favorable channel conditions will decode messages for other users as well. Authors in [54] considered relay implementation while implementing NOMA in a wireless network. In [55-57], authors focused on reducing the pair-wise error probability (PEP). A new strategy was proposed to achieve high coding gains for NOMA relay channel in [58]. In [59], Rayleigh fading channels were used to study the achievable average rate using NOMA with the relaying network. A cooperative relaying system using NOMA is introduced in [60] to enhance the spectrum efficiency. In [31], users are randomly placed to investigate wireless power transfer in NOMA systems. An advanced cooperative wireless power transfer for NOMA is proposed where nearby users (near to source) act as a relay for energy harvesting to support users at further distance.

NOMA Multiple Relay Channel: NOMA with multiple relay channel is considered in the literature. In [51,61], authors considered a multi-terminal network that consists of $K$ independent users that are attempting to access destination via $M$ independent relays. The outage achievable rate is derived for the NOMA with multiple relay channel. Authors in [62] investigated the advantages of joint network and channel coding for relay channel. According to a joint network-channel coding concept, the intermediate nodes of the network can alternatively work as a sender and a receiver.

\section{Non-Cooperative NOMA}

Unlike cooperative NOMA, non-cooperative NOMA does not have any information about the wireless network available prior to transmission. Non-cooperative NOMA is extensively studied in the literature, here we will discuss most common schemes. 
Multi-user shared access (MUSA): MUSA is one of the multiple access schemes in 5G communication systems. MUSA operates on the characteristics of advanced SIC at the receiver end and good spreading sequences [63-65]. Data of all the users is spread with the help of a unique spreading sequence. The data of each user after spreading is then overlapped for transmission. In order to retrieve the data packet of each user, SIC is performed at the receiver end.

Sparse code multiple access (SCMA): SCMA is a frequency domain NOMA scheme which utilizes sparse spreading sequences to improve spectrum efficiency. SCMA scheme takes advantage of low-density signatures (LDS)-OFDMA; however, mapping and spreading are jointly considered in SCMA $[66,67]$. In SCMA, different sparse codewords are used for direct mapping of bit streams. A multi-dimensional constellation is generated in SCMA by using different codewords for each user from dedicated code books. The performance improvement of SCMA comes because of the shaping gain of the multi-dimensional constellation.

Outer loop link adaptation (OLLA): Authors in [43] discussed the gains of NOMA over OMA (i.e., OFDMA) while considering the link adaptation of LTE radio interface. In addition to the dynamic multi-user power allocation which is the functionality of NOMA, adaptive modulation and coding (AMC), hybrid automatic repeat request (HARQ), time/frequency-domain scheduling, and outer loop link adaptation (OLLA) are also incorporated. In [13], authors showed that NOMA's performance is better than that of OMA under multiple configurations. Performance analysis of NOMA is presented in terms of throughput and fairness.

Cognitive radio inspired NOMA (CR-NOMA): In order to enhance the performance gains in $5 \mathrm{G}$ systems, there is a need to incorporate cognitive radio $(\mathrm{CR})$ techniques with NOMA system. In [68], a down link and up link transmission framework in MIMO-NOMA is designed. Authors proposed a CR inspired power allocation scheme to evaluate the performance of MIMO-NOMA. Authors have also stressed that the choice of the power allocation coefficients is very important in order to attain tradeoff between throughput and fairness NOMA. Unlike the fixed power allocation strategy, the CR inspired power allocation scheme ensures the quality-of-service (QoS) requirements of users are met instantaneously. 
Pattern division multiple access (PDMA): Non-orthogonal patterns are designed in PDMA such that diversity is maximized and multiple user overlap is minimized [26]. In PDMA, multiple users are differentiated by the non-orthogonal characteristic pattern in single or multiple signal domains, e.g., power domain, space domain, and code domain [25]. SIC amenable detection at the receiver can help multiple users to get the equivalent degree of diversity. PDMA is a good choice to strike a balance between diversity degree and multiplexing, and can be applicable to various scenarios which include macro-cell coverage, hotspot, indoor access, and M2M communications.

Low density spreading (LDS): Multi-carrier low-density spreading multiple access (MCLDSMA) is a reassuring multiple access strategy which allows optimum multi-user detection. Symbol of every user is dispersed on a small set of sub-carriers where each sub-carrier is shared by multiple users. The distinct design of MC-LDSMA which is very challenging, differentiates the radio resource allocation from the well-known multiple access techniques. MC-LDSMA system is investigated for a single-cell system with the radio resource allocation is studied in [69]. Initially, for a single-user, the optimal power allocation and sub-carriers partitioning schemes are derived. Later, an optimal solution is provided to maximize sum-rate. Simulation results are presented to analyze the performance of the proposed algorithms which show that the presented algorithm outperforms the conventional static resource allocation drastically. Moreover, it is concluded that MC-LDSMA has better spectral efficiency and fairness when compared with OFDMA.

Topological interference management NOMA (TIM-NOMA): The efficiency of the networks and the minimized system overhead are critical in wireless networks especially when the user density is increased with high data rate demand. In [70] the achievement of the optimal degree of freedom (DoF) is discussed. Authors have focused on topological interference management (TIM) and blind interference alignment (BIA) assuming that transmitter has no CSI. This assumption is made to minimize the network overhead. In addition to that, an unusual assignment of multiple access that emerged from NOMA resulted in achieving high gains. For single-inputsingle-output a TIM-NOMA scheme is proposed where $T$ represents the groups in which users are divided and DoF calculated for every user is 1/T. A two-step decoding method is established for 
this scenario. Initially, for the interference caused by considering TIM principle in "inter-group" and then for "intra-group" which will be handled by SIC. Authors have simulated in power domain and results have shown that there is a remarkable improvement in terms of data rate as compared to the conventional methods.

\subsection{User Grouping and Power allocation in NOMA}

In [33], authors presented a cell throughput maximization problem under the constraint of transmission power for both up-link and down-link NOMA systems. Authors proposed a two step methodology that comprises of user grouping followed by optimized power allocation for each group. Authors in [32] proposed a dynamic user grouping and power allocation for NOMA with successive interference cancellation (SIC) in down link MIMO cellular systems. The objective is to maximize overall cell capacity subject to the constraints on transmit power, data rate requirement, and received power. For both $[32,33]$, simulation results are presented to highlight the advantages of NOMA in terms of spectrum efficiency. In [34], authors proposed a user grouping scheme based on their location to minimize interference in a visible light communication based 5G network. Further, authors optimized power allocation for each cell to maximize sum rate subject to the constraint on Quality of Service (QoS). A multi-user grouping with low-complexity and a power allocation scheme is presented by authors in [71] to enhance the performance of group users. It is concluded that for the proposed scheme, there exists a trade-off between computational complexity and user fairness. In [35], authors proposed a cooperative game theoretic approach for user grouping in order to enhance sum rate. This scheme divides the users into several groups and then assigns the time slots to each group resulting in notable improvement in sum rate. Authors in [36] formulated a problem for sum rate maximization over frequency selective fading channel by pairing users corresponding to their channel powers. In addition, a divide-and-allocate approach is proposed for power allocation where users are divided in two groups to apply closed form power allocation solution and this process continues until power is allocated to all users. In [37], a user grouping 
scheme is proposed for down link NOMA while considering the channel correlation between users and the channel gain. Further, pre-coding matrix is optimized to maximize the sum-rate. Authors in [38] proposed a user grouping scheme and decoding order setting in SIC for down link NOMA. The objective is to schedule multiple users per resource block optimally. The proposed scheme offers low complexity for the SIC process as the number of decoding signals are equal to the number of users in each group. However, achievable throughput is also decreased because the proposed scheme considers instantaneous fading conditions during user grouping process.

In summary, the above user grouping schemes do not consider energy harvesting to enhance the energy efficiency in NOMA systems.

Related Work on NOMA with Wireless Power Transfer. p1cm p $1 \mathrm{~cm} \mathrm{p} 3 \mathrm{~cm}$ p $0.5 \mathrm{~cm}$ p $0.5 \mathrm{~cm}$ p $0.5 \mathrm{~cm} \mathrm{p} 7 \mathrm{~cm}$

\section{Ref. Year Objective $\mathbf{U}^{1} \mathbf{E}^{2} \mathbf{P}^{3}$ Remarks}

[33] 2016 Uplink and downlink sum rate maximization $\checkmark \checkmark$ Authors presented a cell throughput maximization problem under the constraint of transmission power for both uplink and downlink NOMA systems.

[32] 2017 Downlink sum rate maximization $\checkmark \checkmark$ Authors proposed a dynamic user grouping and power allocation for NOMA with successive interference cancellation (SIC) in downlink MIMO cellular systems.

[34] 2017 Sum rate maximization $\checkmark \checkmark$ Authors proposed a user grouping scheme based on their location to minimize interference in visible light communication based $5 \mathrm{G}$ network.

[71] 2016 Average throughput $\checkmark \checkmark$ A multi-user grouping with low complexity and a power allocation scheme is presented by authors to enhance the performance of group users.

[35] 2016 Sum rate maximization $\boldsymbol{V}$ Authors proposed a cooperative game theoretic approach for user grouping in order to enhance sum rate.

\footnotetext{
${ }^{1}(\mathrm{U})$ ser grouping.

${ }^{2}(\mathrm{E})$ nergy harvesting.

${ }^{3}(\mathrm{P})$ ower allocation.
} 
[36] 2016 Sum rate maximization $\checkmark \mathfrak{\checkmark}$ Authors formulated a problem for sum rate maximization over frequency selective fading channel by pairing users corresponding to their channel powers. [37] 2015 Sum-rate maximization $\checkmark$ A user grouping scheme is proposed for downlink NOMA while considering the channel correlation between users and the channel gain.

[38] 2015 Optimal user scheduling $\checkmark$ Authors proposed a user grouping scheme and decoding order setting in SIC for downlink NOMA.

[39] 2017 Outage probability $\checkmark \checkmark$ Authors have considered a cooperative NOMA network in which energy harvesting relay is utilized as a medium of communication between users.

[72] 2017 Sum data rate $\checkmark \checkmark \checkmark$ Authors have proposed two cooperative spectrum sharing (CSS) algorithms while utilizing the concept of both time-switching and power-splitting based energy harvesting.

[40] 2016 Sum rate $\checkmark$ An energy harvesting-based cooperative NOMA system is considered, in which one node simultaneously sends independent signals to a stronger node and a weaker node.

[41] 2016 Data rate $\checkmark \checkmark$ A NOMA scheme for the uplink of wireless powered communication networks (WPCNs) is proposed.

[30] 2016 Throughput $\checkmark$ Authors optimized the data rate and fairness improvement for a wireless-powered system with NOMA.

[42] 2016 Sum rates optimization $\checkmark$ Authors have investigated a wireless-powered uplink communication system with NOMA in order to optimize data rate and increase fairness.

[31] 2016 outage probability $\checkmark$ Authors have investigated a NOMA with wireless power transfer in which users are spatially randomly located.

Current work Throughput maximization $\checkmark \checkmark \checkmark$ We investigate joint user grouping, power allocation, and time allocation for NOMA with RF energy harvesting. 


\subsection{Energy Harvesting and Wireless Power Transfer in NOMA}

In [39], authors have considered a cooperative NOMA network in which energy harvesting relay is utilized as a medium of communication between users. Fixed power allocation NOMA and cognitive radio based NOMA are studied for their impact on simultaneous information and power transfer. It is concluded that two NOMA power allocation policies have trade-off among reliability, user fairness, and system complexity. Authors in [72] have proposed two cooperative spectrum sharing algorithms while utilizing the concept of both time-switching and power-splitting based energy harvesting. A set of secondary transmitters which are energy constrained are dependent on energy harvesting. The secondary transmitter acts as a relay to forward primary user symbol and also to get access to channel according to the concept of NOMA simultaneously with primary user. Authors studied optimal energy harvesting ratio that maximizes the sum data rate of both protocols. In [40], an energy harvesting-based cooperative NOMA system is considered, in which one node simultaneously sends independent signals to a stronger node and a weaker node. It is considered that the link between source node and weaker node is not able to satisfy the QoS requirements. Therefore, the stronger node acts as an energy harvesting relay for the weaker node as it has prior knowledge about it based on NOMA approach. The objective is to maximize rate subject to the constraints on QoS and power. A NOMA scheme for the uplink of wireless powered communication networks is proposed in [41]. Authors jointly optimize the BS transmit power and energy harvesting/information transfer duration to maximize the rate region. The proposed approach achieved higher data rates compared to fixed power NOMA in addition to keeping the high level of fairness. In [30], authors optimized the data rate and fairness improvement for a wireless-powered system with NOMA. Two types of decoding order strategies are proposed for a NOMA system: fixed decoding order and time sharing. Also, an efficient greedy algorithm is proposed which is suitable for the practical implementation of the time-sharing strategy. In [42], authors have investigated a wireless-powered uplink communication system with NOMA in order to optimize data rate and increase fairness. In $[31,73]$, authors have investigated a NOMA with wireless power transfer in which users are spatially randomly located. A protocol is designed in 
a way that the NOMA users which are close to the source act as energy harvesting relays to provide power to users at distance. Authors proposed three user selection schemes while considering users distance from the base station and compared their results in terms of outage probability and throughput.

Given the benefits of energy harvesting in NOMA, it is important to investigate user grouping, power allocation, and time allocation to enhance the performance of NOMA systems in more realistic cases. 


\section{Chapter 3}

\section{System Model and Problem Formulation}

\subsection{Network Model}

We consider a network that consists of $K$ users and $N$ resource blocks as shown in Fig. 3.1. It is assumed the number of users is greater than the number of resource blocks, i.e., $K>N$. We assume that each frame with length one is divided into two time slots [42]. In the first time slot, the base station transmit power beacon to the users such that the users can harvest energy for uplink transmission. The amount of time for energy harvesting is set to $1-T$ and time for information transfer is set to $T$ such that $0 \leq T \leq 1$. It is challenging to associate resource block to the users and determine the time sharing between energy harvesting and information transfer such that their required rate is satisfied. Therefore, we use NOMA to accommodate $K$ users in $N$ resource blocks while satisfying user rate requirements. Let $g_{k}$, and $\eta$ be the channel gain of the $k$-th user to the base station (contains path loss, antenna factors etc.) and energy harvesting efficiency, respectively. We assume that the total energy harvested in 1-T duration for the $k$-th user is the energy that is used for transmission in time $T$. Let $P_{B S}$ be the amount of base station transmission power at the first time slot then the harvested energy by the $k$-th user will be $E_{k}=\eta g_{k} P_{B S}(1-T)$ Then, the uplink transmit power of the $k$-th user is

$$
P_{k}=\frac{E_{k}}{T}=\Delta g_{k} \eta P_{B S}
$$


where $\Delta=\frac{1-T}{T}$ and $E_{k}$ is the energy harvested by the $k$-th user.

The base station will use SIC for decoding. Rate of each user depends on its decoding order. We assume that users are sorted according to their channel gain such that $g 1>g_{2} \ldots \ldots . . g_{k}$. Let there be only one resource block and $K$ users then the rate for first user will be

$$
R_{1}=T \log _{2}\left(1+\frac{P_{1} g_{1}^{2}}{N_{0}+\sum_{i=2}^{K} P_{i} g_{i}^{2}}\right),
$$

where $N_{0}$ is the noise spectral density. After some mathematical calculations, it can be written as (Appendix ?? for details):

$$
R_{1}=T \log _{2}\left(1+\frac{\Delta \rho g_{1}^{2}}{1+\Delta \rho \sum_{i=2}^{K} g_{i}^{2}}\right)
$$

The rate for $k$-th user can be written as,

$$
R_{k}=T \log _{2}\left(1+\frac{\Delta \rho g_{k}^{2}}{1+\Delta \rho \sum_{i=k+1}^{K} g_{i}^{2}}\right),
$$

where $\rho=\frac{\eta P_{B S}}{N_{0}}$. It is evident from (3.2) and (4) that the user 1 will face interference from all other $K-1$ users. The $k$-th user, due to SIC will face interference from $K-k$ users and so on. Thus, the rate of user $k$ can be written as

$$
R_{k}=T \log _{2}\left(1+\Delta \rho g_{k}^{2}\right) .
$$

A detailed list of symbols and their description is provided in Table 3.1.

\subsection{Problem Formulation}

In this thesis, the goal is to maximize the system throughput. It is important to note that total throughput can be maximized by considering the minimum data rate requirement of individual users. Thus, we consider a constraint on minimum data rate requirement of individual users. An individual user can use $r$-th resource block for data transmission. We define a binary indicator function $x_{r}^{k}$ that defines usage of $k$-th user for $r$-th resource block. $x_{r}^{k}=1$ in case, when $k$-th user 

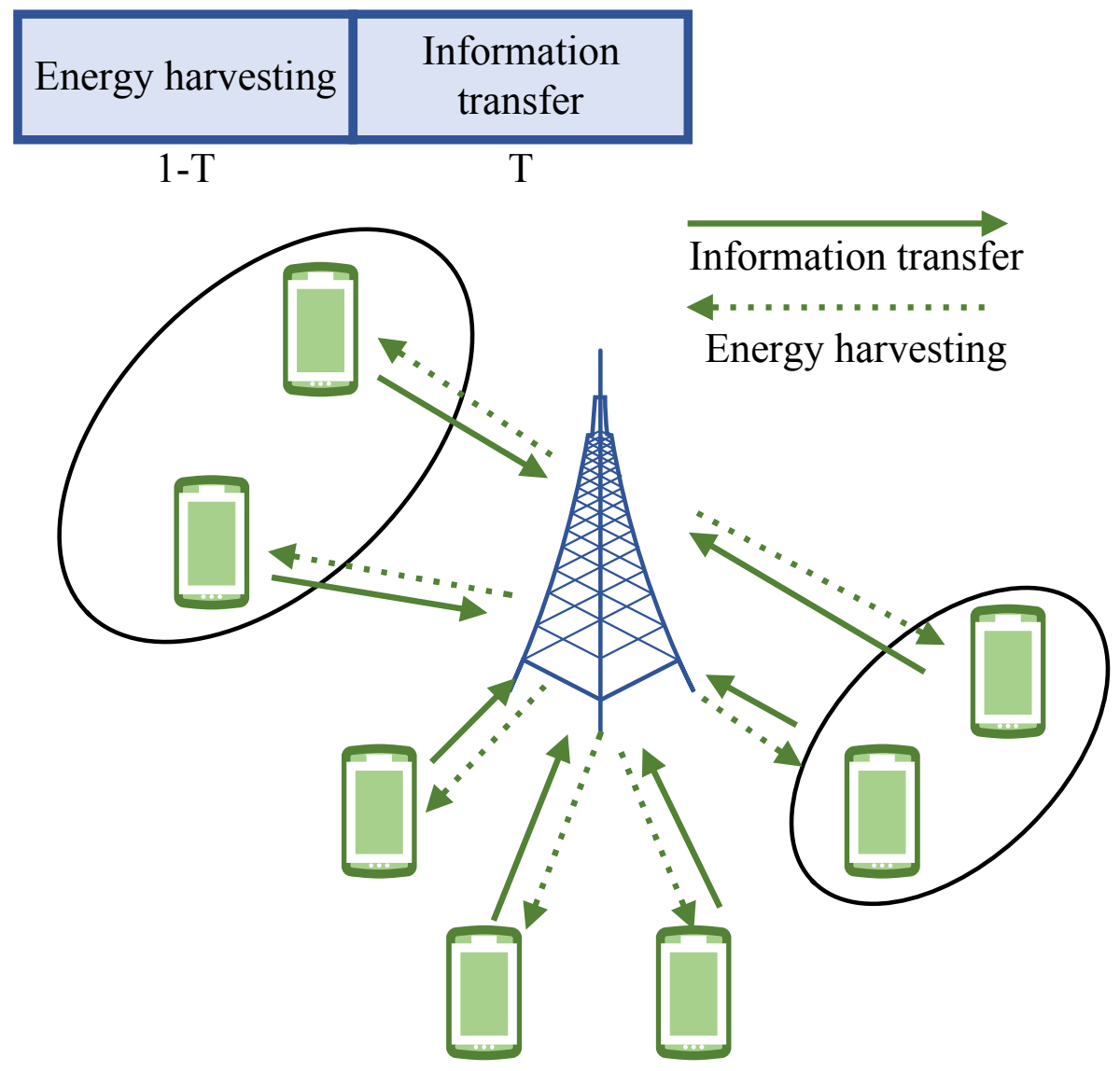

Figure 3.1: An illustration of network that consists of $K$ users and $N$ resource blocks for NOMA with wireless power transfer.

is using $r$-th resource block and 0 otherwise. This can be represented as:

$$
x_{r}^{k}= \begin{cases}1, & \text { if } k \text {-th } \text { user is using } r \text {-th resource block } \\ 0, & \text { otherwise. }\end{cases}
$$

The individual user can either be selected for data transmission or not. A binary indicator function $y_{k}$ defines user selection where $y_{k}=1$ when $k$-th user is selected and 0 otherwise. This can be written as:

$$
y_{k}= \begin{cases}1, & \text { if } k \text {-th } \text { user is selected } \\ 0, & \text { otherwise }\end{cases}
$$


Table 3.1: Description of the symbols used in the model.

\begin{tabular}{ll}
\hline Symbol Description \\
\hline$K$ & Number of users \\
$N$ & Number of resource blocks \\
$T$ & Sharing time of the resource block \\
$P_{B S}$ & Power of the Base station \\
$P_{k}$ & Uplink transmit power of the $k$ - $t h$ user \\
$R_{k}$ & Data rate of $k$-th user \\
$R_{k}^{\text {min }}$ & Minimum data rate of the $k$-th user \\
$g_{k}$ & Channel gain for $k$ - $t h$ user \\
$\eta$ & Energy harvesting efficiency \\
$x_{r}^{k}$ & Usage of $k$-th user for $r$ - $t h$ resource block \\
$y_{k}$ & User selection \\
$\Psi_{i}$ & Set of finite points where the objective function is to be evaluated \\
\hline
\end{tabular}

The objective is to maximize the data rate by optimizing the $X, Y, T_{r}$, and $P_{B S, r}$, where $X$ is the assignment matrix which consists of $\mathrm{x}_{r}^{k} \forall k$ and $r, Y$ is the user selection vector that consists of $\mathrm{y}_{k} \forall k, T_{r}$ is the time sharing in each resource block, and $P_{B S, r}$ is the power in each resource block. The data rate of each user must meet minimum data rate requirement, i.e., $R_{k}^{\min }$ in order to meet QoS requirements. However, practically it may not be possible to meet rate requirement due to transmit power constraint. In this thesis, we maximize data rate by dynamic user grouping, power allocation, and time allocation while satisfying constraints on user throughput and power in each resource block. The utility function $U$ which is equivalent to system throughput for NOMA with RF energy harvesting can be stated as follows:

\section{Given:}

- Total number of users $(K)$

- Total number of resource blocks $(N)$

- Minimum QoS requirement of $k$-th user $\left(R_{k}^{\min }\right)$

- Maximum base station power $P_{B S}^{M A X}$

\section{Objective:}




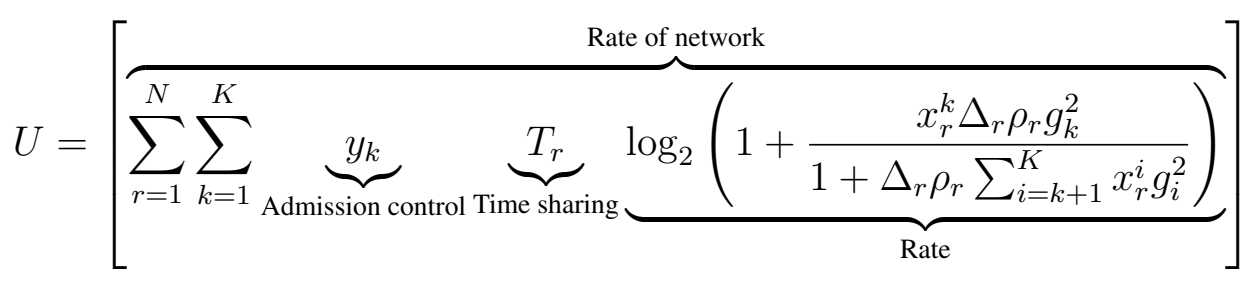

- Maximize system throughput

\section{Determine:}

- Assignment matrix $X$

- User selection vector $Y$

- Time sharing for all $r$-th resource blocks $T_{r}$

- Power in $r$-th resource block $P_{B S, r}$

The utility function for the proposed framework for NOMA with RF energy harvesting can be formulated as in (3.8). The data rate maximization problem can be formulated as:

$$
\max _{X, Y, T_{r}, P_{B S, r}}: U
$$

\section{Subject to}

$$
\begin{aligned}
& C 1: \underbrace{\sum_{r=1}^{N} x_{r}^{k} \leq 1, \forall k} \\
& \text { User resource block assignment } \\
& C 2: \underbrace{\sum_{r=1}^{N} T_{r} \log _{2}\left(1+\frac{x_{r}^{k} \Delta_{r} \rho_{r} g_{k}^{2}}{1+\Delta_{r} \rho_{r} \sum_{i=k+1}^{K} x_{r}^{i} g_{i}^{2}}\right) \geq y_{k} R_{k}^{\text {min }},}_{\text {Rate constraint of selected user }} \\
& C 3: \underbrace{\sum_{r=1}^{N} P_{B S, r} \leq P_{B S}^{M A X}}_{\text {Power budget constraint }} \\
& C 4: \quad \underbrace{x_{r}^{k} \leq y_{k}, \forall k, r} \\
& \text { User assignment/ selection couple constraint } \\
& C 5: y_{k} \in\{0,1\}, x_{r}^{k} \in\{0,1\}, T \in[0,1], P_{B S, r} \geq 0, \forall k, r
\end{aligned}
$$


where $\mathrm{C} 1$ ensures one user cannot use more then one resource block, $\mathrm{C} 2$ grantees minimum data rate requirement of selected user for all resource blocks where $\Delta_{r}=\frac{1-T_{r}}{T_{r}}, \mathrm{C} 3$ ensures power of base station should be less than maximum power for all resource blocks, $\mathrm{C} 4$ assures that $x_{r}^{k}$ should be zero if the $k$-th user is not selected.

The problem in (4.1) is a mixed integer non-linear programming problem (MINLP) since both utility function and constraints have logarithmic terms. Thus, the problem in (4.1) is MINLP which is generally NP-hard. 


\section{Chapter 4}

\section{Proposed Scheme and Comparison}

We use two approaches to solve the problem given in (4.1): 1) optimal solution using exhaustive search algorithm (ESA) and 2) mesh adaptive direct search (MADS) algorithm. We now describe the detailed operations of both approaches.

\subsection{Optimal Solution}

In this section, we will provide details of optimal solution obtained using ESA to solve (4.1). The steps of the ESA are shown in Algorithm 1. This method enumerates all possible combinations of $X$ and $Y$. Hence, we achieve the optimal solution, however, at the cost of computational complexity.

\subsubsection{User Grouping}

The algorithm takes number of users $(K)$, number of resource blocks $(N)$, minimum data rate requirement $\left(R_{k}^{\min }\right)$ as an input. We initialized parameter $D$ as number of discrete variables which is equal to $K+K$ and initial best solution as 0 , i.e., we are looking for the best solution. During the execution phase, which runs for $2^{D}$ iterations all values of $X$ and $Y$ are evaluated to find best solution for (4.1). In each iteration, a binary vector is initiated and corresponding values are saved 
in $Z$. In the next steps, the functions $\operatorname{get} x()$ and $\operatorname{gety}()$ extract the association matrix $X$ and user selection vector $Y$ from the $Z$ and stores corresponding values in $X$ and $Y$, respectively. Then, we check the feasibility of $X$ and $Y$ to avoid evaluation of objective function using infeasible solution. The pseudo code to check feasibility of $\mathrm{X}$ and $\mathrm{Y}$ is given in Algorithm 2. $\mathrm{X}$ and $Y$ will produce infeasible solutions if 1) no user is selected, or 2) $k$-th user is not selected and its corresponding assignment variables are non-zero, or 3) $k$-th user is selected and none of its corresponding variable are non-zero. For better understanding, let $K=3$ and $N=2$ which results in $D=9$ discrete variables, i.e., $\left[x_{1}^{1} x_{1}^{2} x_{1}^{3} x_{2}^{1} x_{2}^{2} x_{2}^{3} y_{1} y_{2} y_{3}\right]$. Then the vectors $X=\left[x_{1}^{1} x_{1}^{2} x_{1}^{3} x_{2}^{1} x_{2}^{2} x_{2}^{3}\right]$ and $Y=$

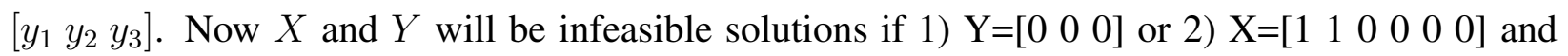
$\mathrm{Y}=\left[\begin{array}{lll}1 & 0 & 0\end{array}\right]$ (user 2 is not selected but its corresponding assignment variable $x_{1}^{2}=1$, or 3) $\mathrm{X}=\left[\begin{array}{lll}0 & 1 & 0\end{array}\right.$ $\left.\begin{array}{lll}0 & 0 & 0\end{array}\right]$ and $\mathrm{Y}=\left[\begin{array}{lll}1 & 0 & 0\end{array}\right]$ (user 1 is selected but none of its corresponding variable is non-zero).

\subsubsection{Time and Power Allocation}

For feasible $\mathrm{X}$ and $\mathrm{Y}$ we solve following non-linear programing (NLP) problem:

$$
\max _{T_{r}, P_{B S, r}}: U
$$

Subject to

$$
\begin{aligned}
& C 2: \underbrace{\sum_{r=1}^{N} T_{r} \log _{2}\left(1+\frac{x_{r}^{k} \Delta_{r} \rho_{r} g_{k}^{2}}{1+\Delta_{r} \rho_{r} \sum_{i=k+1}^{K} x_{r}^{i} g_{i}^{2}}\right) \geq y_{k} R_{k}^{\text {min }},}_{\text {Rate constraint of selected user }} \\
& C 3: \underbrace{\sum_{r=1}^{N} P_{B S, r} \leq P_{B S}^{M A X}}_{\text {Power budget constraint }}, .
\end{aligned}
$$

Algorithm will update BestSolution if the solution obtained is better than the one in previous iterations. Finally, at the end of $2^{D}$ iterations, the output of the algorithm is BestSolution which represents the optimal solution of problem in (4.1). 


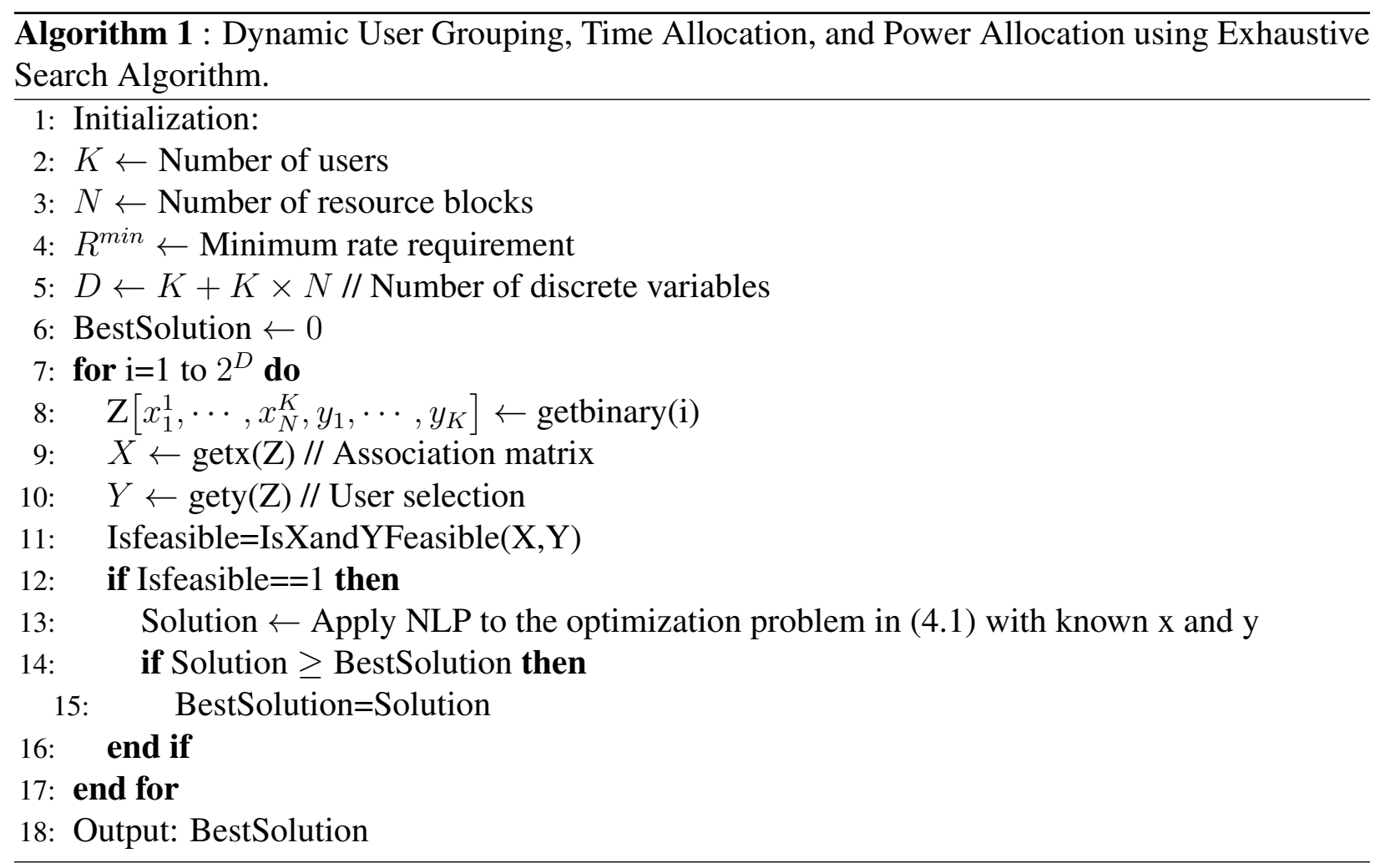

\subsubsection{Mesh Adaptive Direct Search Algorithm}

We adopted mesh adaptive direct search (MADS) algorithm to obtain the sub-optimal solution of problem given in (4.1). The MADS algorithm is usually a solution for non-linear optimization problem. The MADS is an extended version of generalized pattern search (GPS) based on polling mechanism. Polling is the local scrutiny of objective function in the space of optimization variables. The MADS is a derivative free procedure and iterative in nature, where $i$-th iteration consists of two steps, i.e., searching and polling. During the search step, if it gets a better solution, then it elongates search space and perform searching step again. When the searching step fails to find a better solution, then polling step invokes and narrows down the search around the current solution. This will lead to the convergence of the algorithm.

Given an iteration $i$, the MADS algorithm generates a current mesh and finite number of trial points. In $i$-th iteration, the current mesh can be defined as: 


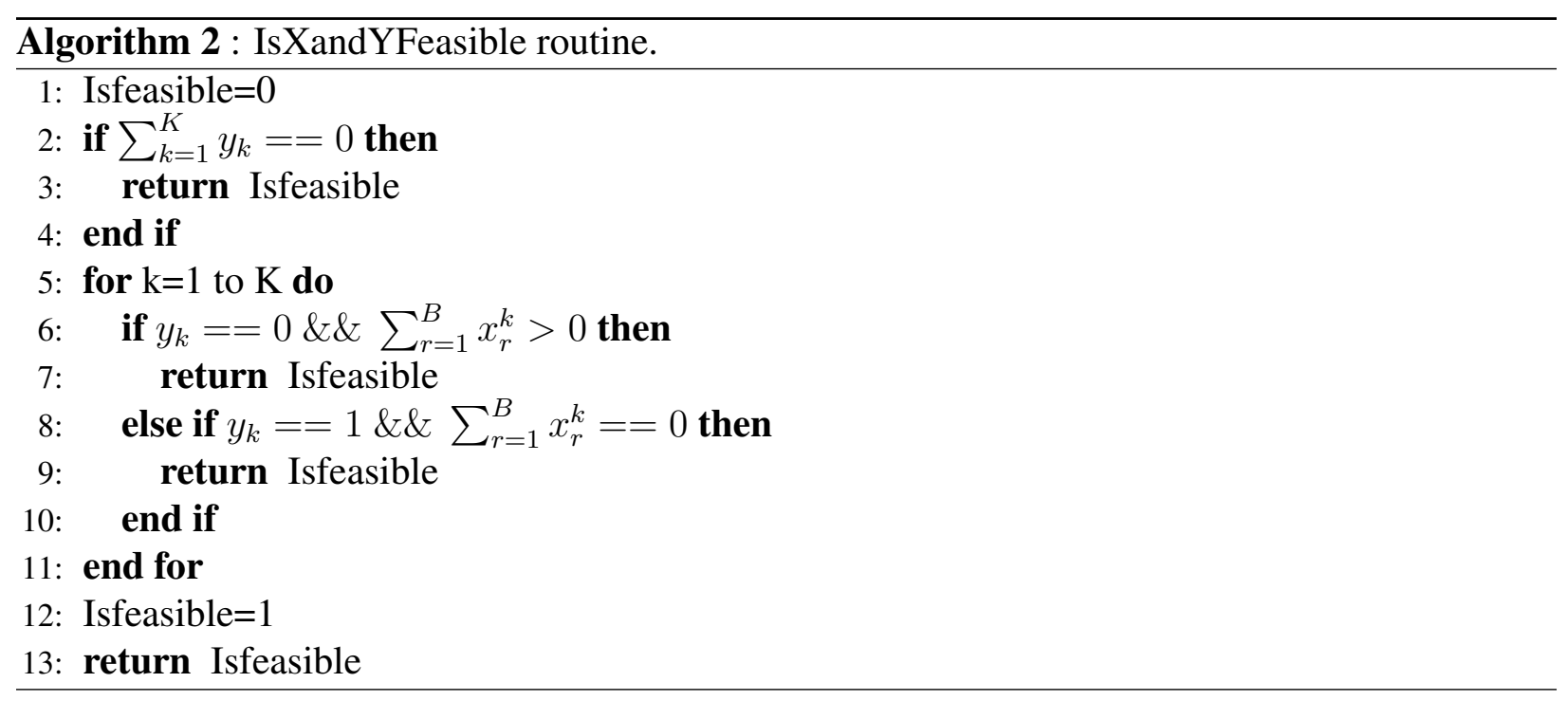

$$
M_{i}=\bigcup_{a \in \Psi_{i}}\left\{a+\nabla_{i, m} D_{i, m}\right\}
$$

where $a$ is any arbitrary starting trial point in the feasible region and $\Psi_{i}$ is the set of finite points where the objective function is to be evaluated, $\nabla_{i, m} \in \mathbb{R}_{+}$is the mesh size parameter, and $D_{i, m}$ is the finite set of mesh directions.

In this thesis, we consider fixed number of trial points in current mesh which are located on the current mesh with four directions (left, right, up, and down) scaled by $\nabla_{i, m}$. Now, in the first step called searching, the objective function values are computed at four mesh points. These values are then compared with the current value which is so far the best solution of objective function in (4.1) to find yet better solution. If a better solution is found at any trial point then the trial point, is called an improved mesh point and iteration is considered as successful iteration. When an improved mesh point is found then it may stop or continue searching for better mesh points. It is worth to mention that the constraints of (4.1) are evaluated to determine feasibility of the solution, and objective function first values are then computed only in the case when constraints are feasible. The next iteration $i+1$ will start with updated incumbent solution and mesh parameter $\nabla_{i+1, m} \geq \nabla_{i, m}$. 


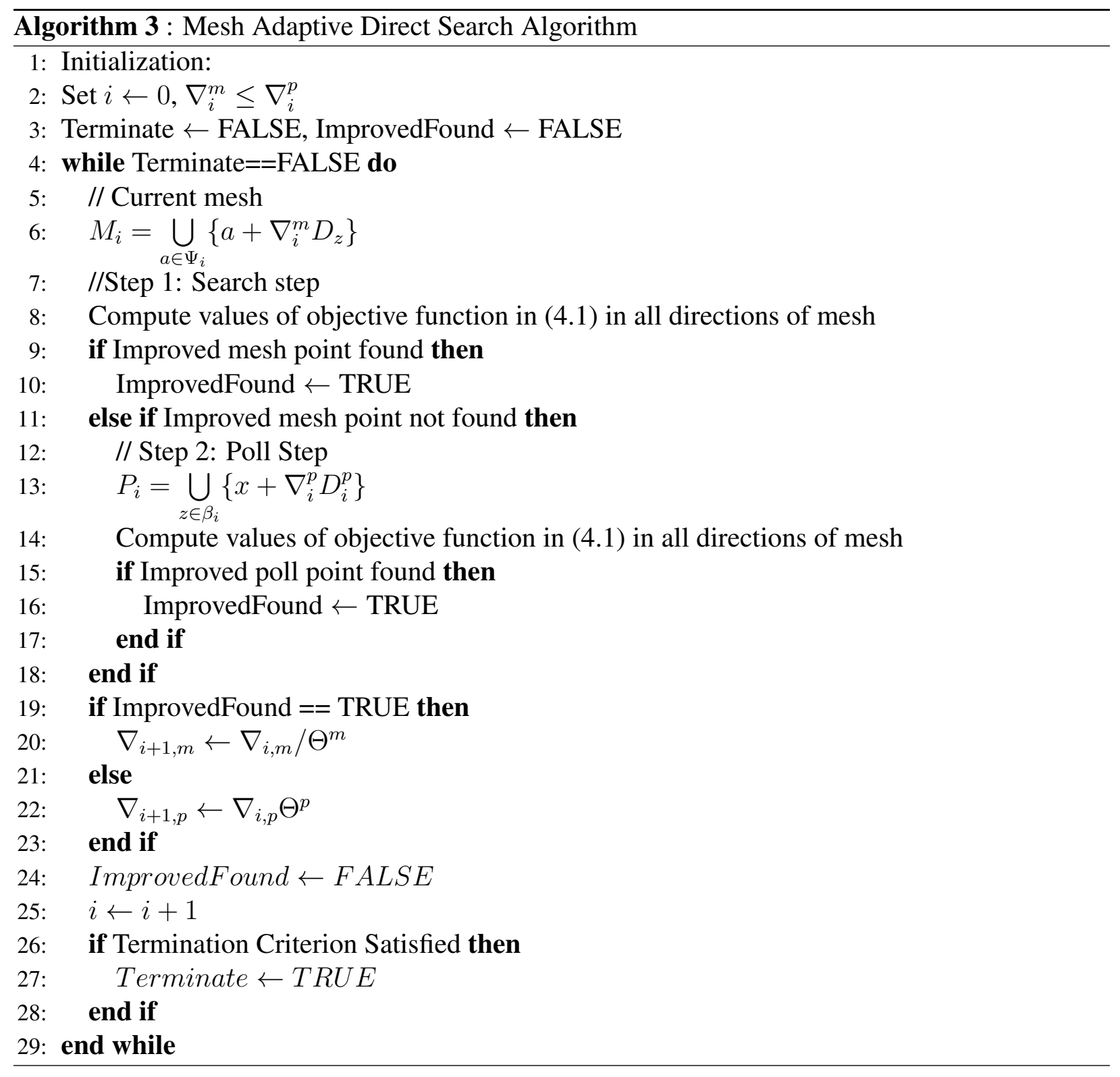

In the case when no improved mesh point is found, then the polling step is invoked. Here, set of poll points is defined as $P_{i}$ with poll size parameter $\nabla_{i, p}$ where $\nabla_{i, m} \leq \nabla_{i, p}$. The objective function is then evaluated at each poll point to find better solution, however, $\nabla_{i+1, p}$ is reduced in case better solution is found and choose the improved point as incumbent solution for next iteration. This will help to further explore better solution in the vicinity of incumbent solution. The key difference between the MADS and GPS is the size parameter. In the case of MADS, $\nabla_{i}^{m} \leq \nabla_{i}^{p}$; whereas in the case of GPS there is only a single parameter $\nabla_{i}=\nabla_{i}^{m}=\nabla_{i}^{p}$. The MADS algorithm to solve 
optimization problem in (4.1) is given in Algorithm 3.

\subsection{Performance Analysis}

In this section, we evaluate the performance of adopted ESA and MADS algorithm. The ESA provides optimal solution and thus is used as a benchmark to compare results of MADS algorithm. We consider the following four scenarios based on number of users $(\mathrm{K})$, number of resource block $(N)$ and minimum data rate requirement $\left(R_{k}^{\min }\right)$ for the joint user grouping, power allocation, and time allocation for NOMA with RF energy harvesting: 1) $K=6, N=2$, and $R^{\text {min }}=125 \mathrm{kbps}$, 2) $K=6, N=2$, and $\left.R^{\text {min }}=1000 \mathrm{kbps}, 3\right) K=5, N=3$, and $R^{\text {min }}=125 \mathrm{kbps}$, and $K=5$, $N=3$, and $R^{\text {min }}=2000 \mathrm{kbps}$. Detailed simulation parameters are given in Table 4.1.

\subsubsection{Comparison between MADS and ESA algorithm}

Fig. 4.1 illustrates comparison for MADS algorithm and ESA for scenario 1. Fig. 4.1 (a) shows resource block index versus user index when the minimum data rate requirement is $125 \mathrm{kbps}$ for each user. It is evident that ESA allocates only user 1 to resource block 1 and rest of all users to resource block 2. On the other hand, MADS algorithm allocate resource block 1 for user 1, 3, and 4 and resource block 2 for user 2 and 6 . However, user 5 is not assigned to any of the resource block since all of the constraints are not satisfied. Fig. 4.1 (b) shows the user power in $\mathrm{dBm}$ for each resource block. It is obvious that the user power is a little more in the case of resource block 2, however, there is not significant difference when MADS algorithm is compared with ESA. Fig. 4.1 (c) shows user rate for each user index. It is clear that each user has equal or higher data rate in the case of MADS when compared with ESA. However, it is important to note that MADS assigned only 5 users out of total 6 users, whereas ESA successfully assigned all 6 users. Fig. 4.1 (d) compares MADS and ESA interms of time sharing for each resource block. It is noticeable that the time shared between transmission and energy harvesting is same for both MADS and ESA in the case of resource block 1 . On the contrary, in the case of resource block 2, more time is allocated 
Table 4.1: Simulation parameters.

\begin{tabular}{ll}
\hline parameter & Value \\
\hline Number of users $(\mathrm{K})$ & $5-6$ \\
$\begin{array}{l}\text { Number of resource blocks } \\
(\mathrm{N})\end{array}$ & $2-3$ \\
Minimum data rate require- & $125 \mathrm{kbps} 2000 \mathrm{kbps}$ \\
ment $\left(\mathrm{R}_{\text {min }}\right)$ & \\
Path loss exponent & 3 \\
$\mathrm{P}_{M A X}$ & $1 \mathrm{e}^{-4} \mathrm{~W}$ \\
\hline
\end{tabular}
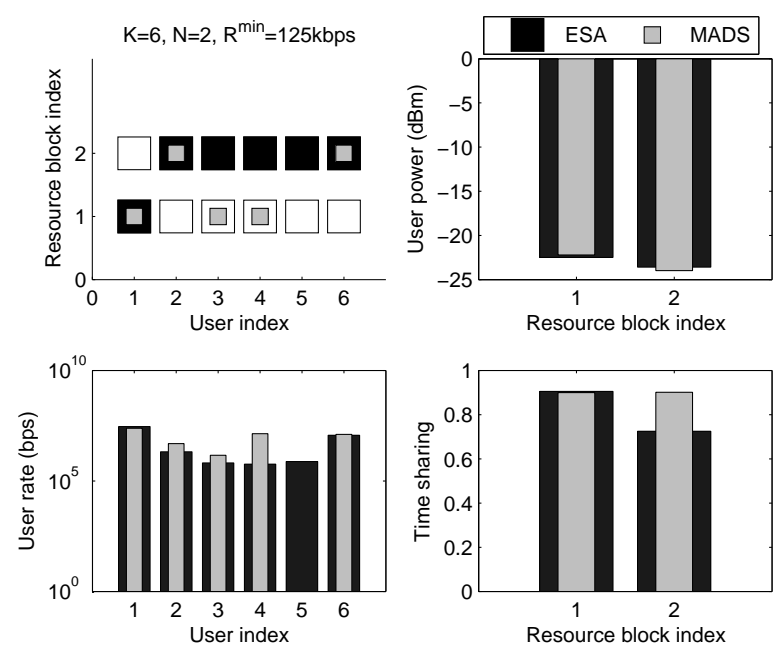

Figure 4.1: Performance comparison of MADS algorithm versus ESA for $\mathrm{K}=6, \mathrm{~N}=2$, and $R^{\text {min }}=125 \mathrm{kbps}$ : (a) resource block index versus user index, (b) user power for each resource block, (c) user rate for each user, and (d) time sharing for each resource block.

for transmission when MADS algorithm is applied in comparison with ESA. This means, in ESA, each user has more time for energy harvesting compared with MADS algorithm.

Fig. 4.2 shows a comparison for scenario 2. Resource block index versus user index is shown in Fig. 4.2 (a) where $K=6, N=2$, and $R^{\text {min }}=1000 \mathrm{kbps}$. In this case ESA allocates user 2, 4, 5 and 6 to resource block 1 and user 1 and 3 to resource block 2. On the contrary, MADS algorithm allocate resource block 1 only to user 5 and resource block 2 to user 1, 2, and 6. User 3 and 4 are not assigned any resource block for this case since all constraints are not satisfied. Fig. 4.2 (b) shows the user power in $\mathrm{dBm}$ for each resource block. It is clear for both resource blocks that ESA assigned more power to meet the data rate requirement when compared to the power assigned by 

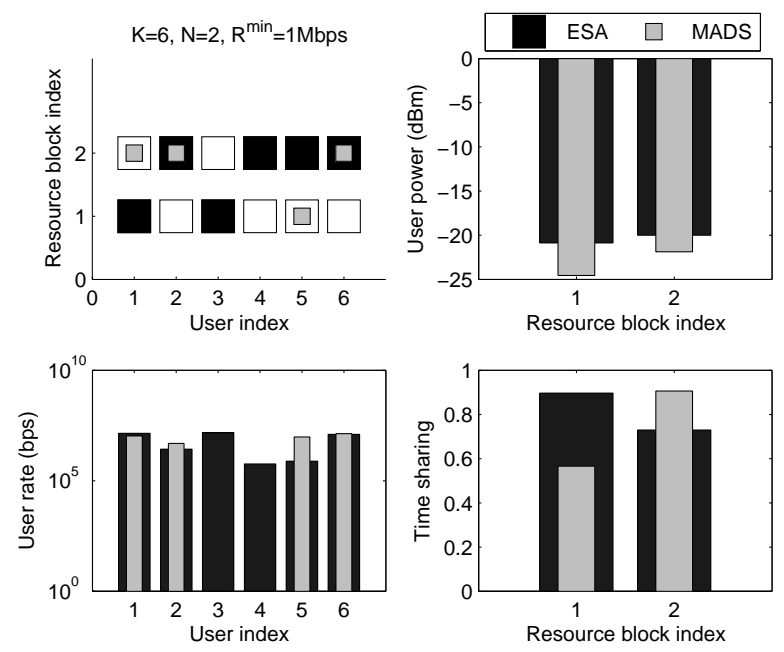

Figure 4.2: Performance comparison of MADS algorithm versus ESA for $\mathrm{K}=6, \mathrm{~N}=2$, and $R^{\text {min }}=1 \mathrm{Mbps}$ : (a) resource block index versus user index, (b) user power for each resource block, (c) user rate for each user, and (d) time sharing for each resource block.

MADS. Fig. 4.2 (c) clearly illustrates that each user has equal or higher user rate in the case of MADS when compared with ESA. However like in scenario 1, ESA was successful in assigning all 6 users to the resource blocks whereas MADS only assigned 4 out of 6 users. Fig. 4.2 (d) provides a comparison for time sharing for both approaches. For resource block 1, MADS gets more time for energy harvesting whereas for resource block 2, MADS allocates more time for transmission as compared to ESA.

Fig. 4.3 is an illustration for scenario 3. $K=5, N=3$, and $R^{\min }=125 \mathrm{kbps}$ are choosen for the resource block index versus user index in Fig. 4.3 (a). In this case, ESA allocates user 3, 4 and 5 to resource block 1 , user 1 to resource block 2 and user 2 to resource block 3 . On the contrary, MADS algorithm allocates user 1 and 5 to resource block 1, user 2 to resource block 2, and user 3 and 4 to resource block 3. Unlike resource blocks 2 and 3, resource block 1 requires more power with MADS as compared to ESA shown in Fig. 4.3 (b). Fig. 4.3 (c) depicts that MADS users rate is better or very similar to when ESA is used. Fig. 4.3 (d) illustrate time sharing for each resource block. For resource block 1, unlike resource block 2 and 3, MADS needs more time for transmission as compared to ESA.

Fig. 4.4 shows a comparison for scenario 4 . Where $K=5, N=3$, and $R^{\text {min }}=2000 \mathrm{kbps}$. 

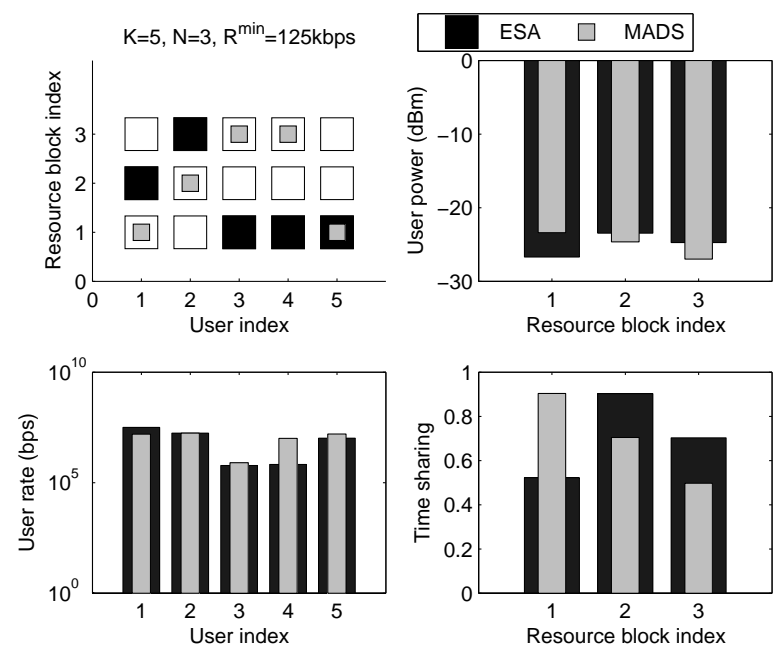

Figure 4.3: Performance comparison of MADS algorithm versus ESA for $\mathrm{K}=5, \mathrm{~N}=3$, and $R^{m i n}=125 \mathrm{kbps}$ : (a) resource block index versus user index, (b) user power for each resource block, (c) user rate for each user, and (d) time sharing for each resource block.
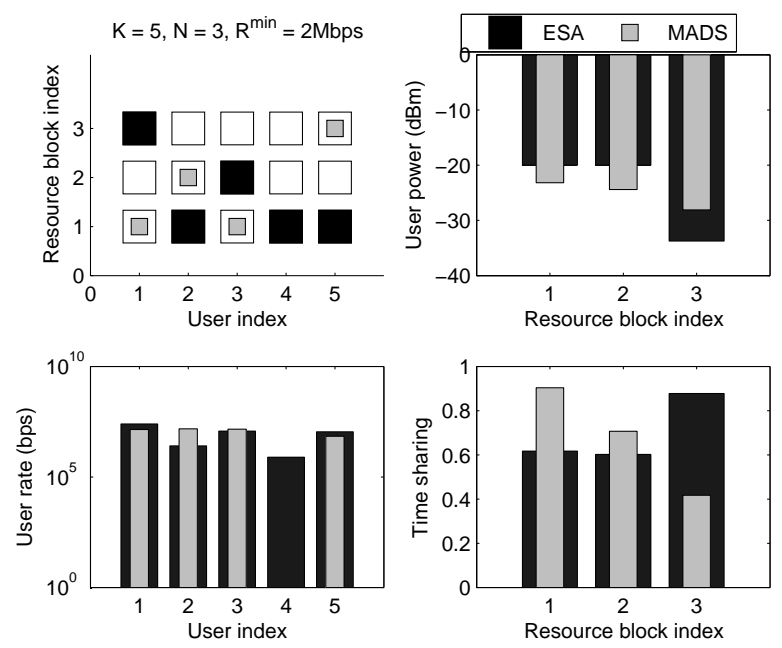

Figure 4.4: Performance comparison of MADS algorithm versus ESA for $\mathrm{K}=5, \mathrm{~N}=3$, and $R^{m i n}=2 \mathrm{Mbps}$ : (a) resource block index versus user index, (b) user power for each resource block, (c) user rate for each user, and (d) time sharing for each resource block. 
Resource block index versus user index is shown in Fig. 4.4 (a). In this case, ESA allocates user 2, 4 and 5 to resource block 1 , user 3 to resource block 2 and user 1 to resource block 3 . Whereas, MADS algorithm allocate user 1 and 3 to resource block 1, user 2 to resource block 2, and user 5 to resource block 3. User 4 is not allocated any resource by MADS since all the constraints are not satisfied. Unlike resource blocks 1 and 2, resource block 3 requires more power with MADS as compared to ESA as shown in Fig. 4.4 (b). It is evident from Fig. 4.4 (c), that with MADS users rate is better or very similar to when ESA is performed. Fig. 4.4 (d) shows time sharing between energy harvesting and information transfer. For resource block 3, unlike resource block 1 and 2, MADS needs less time for transmission as compared to ESA. Which also means that resource block 3 will get more time to harvest energy in MADS as compared to ESA.

\subsubsection{Scalability}

Here, we will discuss the scalability of MADS algorithm. We provide results for three scenarios illustrated as follows: 1) $K=24, N=2$, and $\left.R^{\min }=125 \mathrm{kbps}, 2\right) K=24, N=1$, and $\left.R^{\text {min }}=1000 \mathrm{kbps}, 3\right) K=24, N=2$, and $R^{\text {min }}=1000 \mathrm{kbps}$. Although ESA provides an optimal

solution and used as a benchmark in Figs. 4.1, 4.2, 4.3, and 4.4. However, the complexity of system increases exponentially when we use ESA for obtaining optimal results. Complexity of both the algorithms is separately explained in section 4.2.3. In order to obtain results when the number of users is increased to 24 with ESA demand plenty of resources. Thus, we provide results for the mentioned scenarios with MADS algorithm only.

Fig. 4.5 illustrates results for scenario 1, i.e., $K=24, N=2$, and $R^{\text {min }}=125 \mathrm{kbps}$. Resource block index versus user index is shown in Fig. 4.5 (a). MADS algorithm allocate user 1, 4, 5, 6, 7, $11,13,14,16,17,20$ and 21 to resource block 1 and user 2, 3, 8, 9, 12 and 24 to resource block 2. User 10, 15, 18, 19, 22 and 23 are not allocated any resource by MADS since all the constraints were not satisfied. Fig. 4.5 (b) is a plot for power allocation. Resource block 1 is allocated more power than resource block 2 as it is accommodating more users. The transmission rate of all the 24 users is shown in the Fig. 4.5 (c). This plot shows that the individual transmission rate and the 

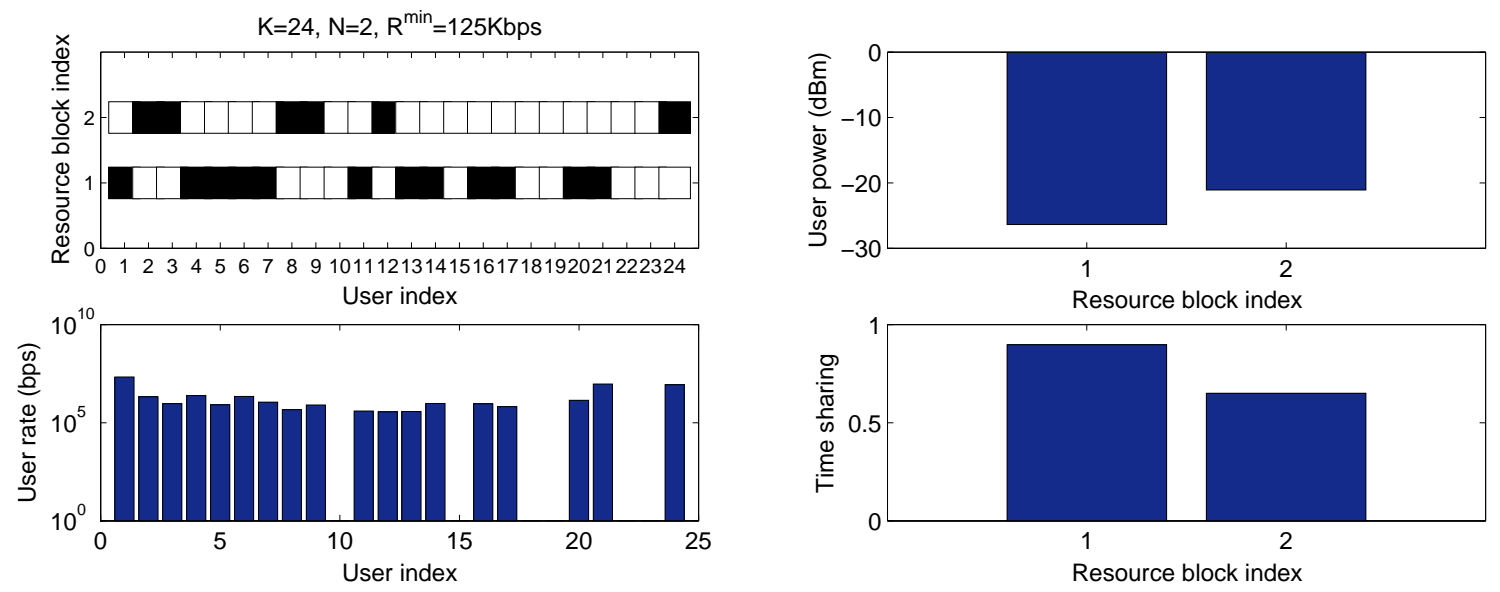

Figure 4.5: Performance of MADS algorithm for $\mathrm{K}=24, \mathrm{~N}=2$, and $R^{\mathrm{min}}=125 \mathrm{kbps}$ : (a) resource block index versus user index, (b) user power for each resource block, (c) user rate for each user, and (d) time sharing for each resource block.

overall throughput of the system has increased tremendously. In Fig. 4.5 (d) time sharing between energy harvesting and information transfer is shown. For resource block 1, unlike resource block 2, MADS needs more time for transmission, which also means that resource block 2 will get more time to harvest energy as compared to resource block 1.

Fig. 4.6 illustrates results for scenario 2, i.e., $K=24, N=1$, and $R^{\text {min }}=1 \mathrm{Mbps}$. MADS only allocated resource block to user 1, 2, 6 and 24 as shown in Fig. 4.6 (a). The rest of the users were not allocated any resource as the constraint requirement did not meet. The plot in Fig. 4.6 (b) shows all the power available in $\mathrm{dBm}$ was allocated to the only resource block for fulfilling the requirements of the assigned users. The data rate of the four assigned users is shown in the plot Fig. 4.6 (c). In Fig. 4.6 (d) shows time sharing. After delicately transferring information of the users whatever time is left with in that time slot is utilized for energy harvesting.

Fig. 4.7 shows a comparison for scenario 3, i.e., $K=24, N=2$, and $R^{\text {min }}=1 \mathrm{Mbps}$. Resource block index versus user index is shown in Fig. 4.7 (a). MADS algorithm allocate resource block 1 to user $1,2,3,4,5,7,8,13,16,17$ and 18 . Whereas resource block 2 is assigned to only user 24. The rest of the users were not assigned any resource block as their constraints requirements were not satisfied. Fig. 4.2 (b) shows the user power in $\mathrm{dBm}$ for each resource block. Resource 

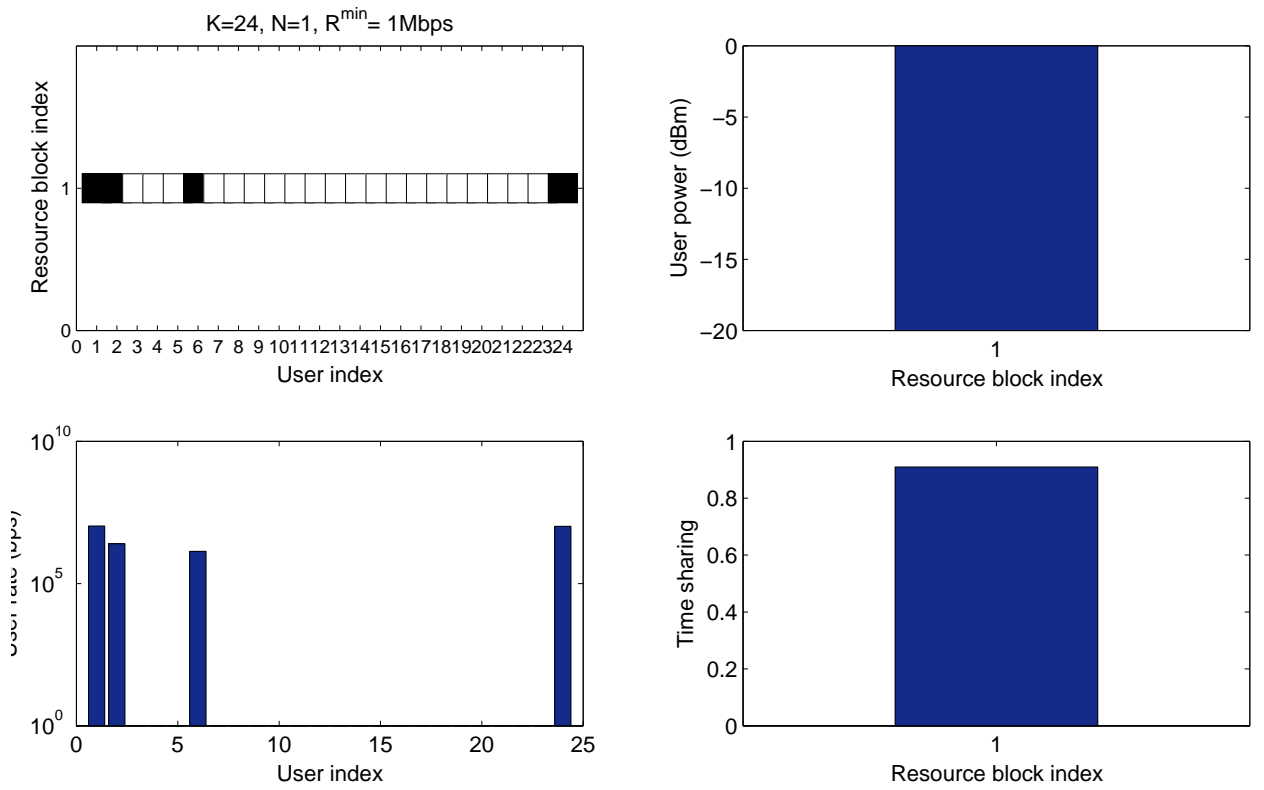

Figure 4.6: Performance of MADS algorithm for $\mathrm{K}=24, \mathrm{~N}=1$, and $R^{\min }=1 \mathrm{Mbps}$ : (a) resource block index versus user index, (b) user power for each resource block, (c) user rate for each user, and (d) time sharing for each resource block.
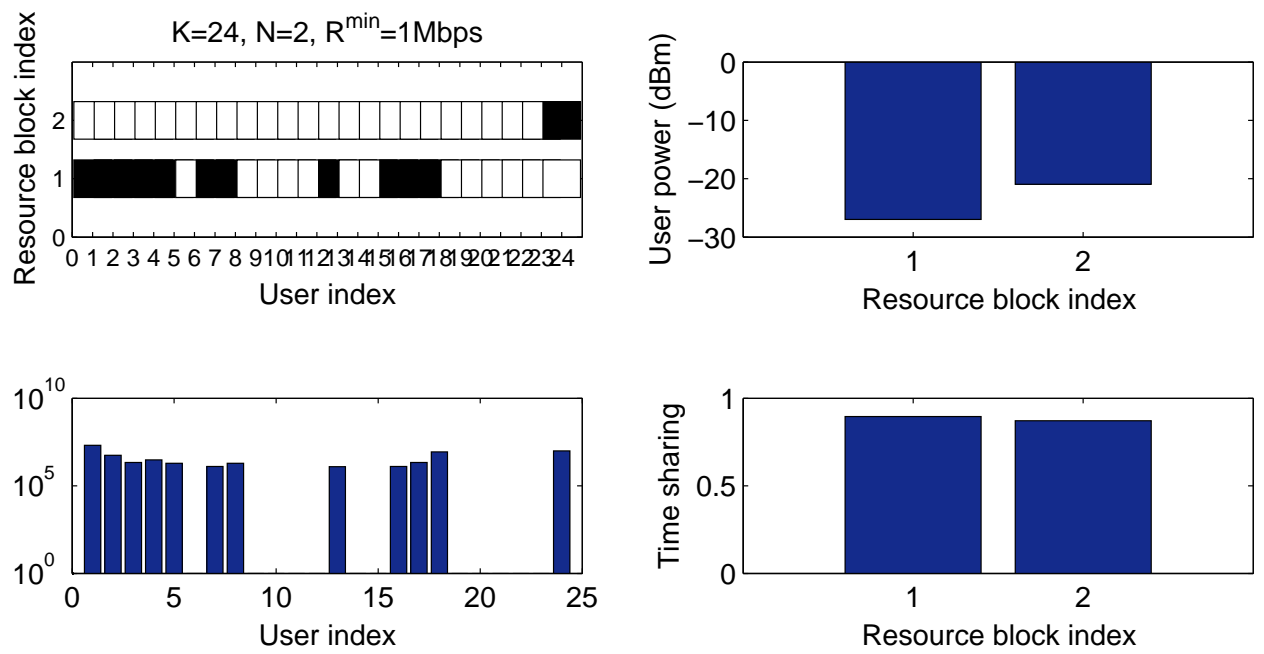

Figure 4.7: Performance comparison of MADS algorithm versus ESA for $\mathrm{K}=24, \mathrm{~N}=2$, and $R^{\text {min }}=1 \mathrm{Mbps}$ : (a) resource block index versus user index, (b) user power for each resource block, (c) user rate for each user, and (d) time sharing for each resource block. 
Table 4.2: Complexity comparison of ESA versus MADS algorithm.

\begin{tabular}{lll}
\hline Parameters & ESA & MADS $(\epsilon=0.001)$ \\
\hline$K=6$ and $N=2$ & 262,144 & 324,000 \\
$K=8$ and $N=2$ & $16,777,216$ & 576,000 \\
$K=6$ and $N=3$ & $16,800,000$ & 576,000 \\
$K=8$ and $N=3$ & $4.2950 \mathrm{e}+09$ & $1,024,000$ \\
\hline
\end{tabular}

block 1 is assigned more power than resource block 2 as it is accommodating more users. In plot of Fig. 4.2 (c) user rate is illustrated for all the users. Fig. 4.2 (d) provides a comparison for time sharing for resource blocks. For resource block 1, the time for information transfer is slightly more than that of resource block 2 .

\subsubsection{Complexity}

The complexity of ESA increases exponentially with the number of users and resource blocks. The complexity of ESA is $O\left(2^{K+K \times N}\right)$. In contrast, the MADS algorithm converges to $\epsilon$-optimal solution in finite number of steps [74,75]. Further, the convergence of MADS algorithm is independent of starting point and converges to global optimal solution with $\epsilon$ error tolerance. The complexity of MADS algorithm is $O\left(\frac{(K+K \times N)^{2}}{\epsilon}\right)$. Therefore, we can achieve a sub-optimal solution using the MADS algorithm for the problem in (4.1) with less complexity when compared to the ESA. In the case of MADS algorithm, some users may not get resources when compared to ESA. This is because of sub optimal solution provided by MADS algorithm. Table 4.2 shows a complexity comparison of ESA versus MADS algorithm for different number of users $(K)$ and resource blocks $(N)$. It is worth to note that complexity of MADS is high for small values of $K$ and $N$, however, MADS clearly outperforms ESA for rest of all cases. 


\section{Chapter 5}

\section{Conclusions and Future work}

\subsection{Conclusions}

A large number of wireless devices demand spectral efficiency and massive connectivity which has shifted the paradigm of orthogonal multiple access to non-orthogonal multiple access. In this thesis, we demonstrated mathematical framework for NOMA with energy harvesting. This framework includes user grouping in their respective resource blocks, time allocation for transmission and energy harvesting, and power allocation. The objective of this framework is to achieve maximum throughput while satisfying minimum data rate requirement of each admitted user and user power in each resource block. The proposed mathematical framework is mixed integer non-linear programming (MINLP). We adopted brute force method (exhaustive search algorithm (ESA)) which provides optimal solution and MADS algorithm which provides $\epsilon$-optimal solution. Four scenarios based on the number of users, number of resource blocks, and minimum data rate are considered for simulation results. User grouping in their respective resource blocks is shown for each scenario. User power for each resource block, user rate for each user, and time sharing for each resource block are also compared for both MADS algorithm and ESA. It is shown that the results for MADS algorithm are near to or equal to the ESA, i.e., optimal solution with less complexity. 


\subsection{Future Research Directions}

Although extensive research in NOMA has been done in recent past, there are areas that need more attention. In this section, we briefly discuss some of these areas.

\subsubsection{Optimal/ Low Complexity Receiver Design}

The NOMA schemes and optimization solutions discussed in this thesis provide solutions for a vast range of challenges such as spectral efficiency, energy efficiency, etc. Nevertheless, the full benefits of NOMA can be achieved with an optimal/ low complexity receiver design. For example, a message passing algorithm can be used for the receiver design in NOMA. However, its complexity is the major hurdle for the massive connectivity in 5G networks. Similarly, the performance of some users may degrade due to error propagation when using SIC-based receivers [7]. Thus, there is a need of optimal/ low complexity receiver design and performance analysis for NOMA to support massive connectivity in 5G networks. There are some novel receiver designs proposed in literature $[76,77]$, however, there is a need for more detailed analysis and explore the capacity gain. Further, it is important to investigate reconfigurable receiver design for accurate detection with less complexity.

\subsubsection{NOMA with imperfect CSI}

Most of the research in NOMA assume perfect CSI can result in significant overhead in practical systems. There is a limited work on NOMA with partial or imperfect CSI. For example, the performance of NOMA with partial channel information is evaluated in [78]. An improved CSI feedback scheme for NOMA is presented in [79] which offers several advantages including user pairing and improved performance. One issue is to study the effect of imperfect CSI on the reliability of NOMA systems. The effect of imperfect CSI on different NOMA schemes and detailed throughput analysis are needed to be done. In addition, many existing NOMA protocols need CSI. This may have an impact on system overhead. Thus, feedback schemes with reduced number 
of bits in NOMA systems can give the robust performance when the interference among users is severe. It is important to design a feedback scheme with an optimal number of bits to achieve required performance with less overhead.

\subsubsection{MIMO-NOMA and Internet of Things}

Internet of things (IoT) is one of the key building blocks of the future wireless networks. The expected growth of IoT devices demands spectral efficiency and massive connectivity. Thus, it is important to investigate joint user allocation and beamforming schemes in MIMO-NOMA to reduce the computation complexity [5]. Further, the MIMO-NOMA small packet transmission schemes are needed to meet diverse QoS requirements in IoT. Recently, a MIMO-NOMA design for small packet transmission in IoT is proposed [80], however, only limited number of scenarios are discussed. More detailed studies with efficient packet transmission schemes are required. In addition, it is of prime importance to address the challenges associated with massive connectivity such as receiver design for low power devices, resource allocation signaling overhead, etc. Also, there is a need to develop prototypes with multi-objective optimal design to demonstrate the impact of MIMO-NOMA on the performance of IoT applications.

\subsubsection{Optimal Wireless Power Transfer}

Wireless power transfer is an efficient solution to deal with energy constrained applications. One of the key benefits of NOMA is that users near to BS (likely to have better channel conditions) can use a channel occupied by users far from BS (likely to have poor channel conditions). [81]. The users near the BS can act as relays to users far from BS to enhance transmission reliability. Thus, wireless power transfer can an efficient solution considering the fact that devices in the network are energy constrained (especially in the case of IoT applications). There are some solutions proposed for the optimal design of NOMA with wireless power transfer $[31,82,83]$. There are still open issues in wireless power transfer in NOMA systems for IoT applications. Further, it is important to investigate other parameters for NOMA with wireless power transfer such as data rate, fairness, 
etc. Therefore, new optimized spectral and energy efficient solutions should be developed for wireless power transfer in NOMA.

In addition, there are some other areas for optimization in NOMA, including, optimal user pairing/ clustering to reduce system complexity and cross-layer optimization to optimizing the parameters from different layers to improve the performance of NOMA. 


\section{Appendix}

\section{Mathematical Calculations to Calculate $R_{1}$}

The rate for the first user in the case of one resource block and $K$ users is given in (3.2) and can be written as:

$$
R_{1}=T \log _{2}\left(1+\frac{\frac{P_{1}}{N_{0}} g_{1}^{2}}{1+\sum_{i=2}^{K} \frac{P_{i}}{N_{0}} g_{i}^{2}}\right)
$$

since $P_{1}=\Delta g_{1}^{2} \eta P_{B S}$

$$
\begin{aligned}
R_{1} & =T \log _{2}\left(1+\frac{\frac{\Delta g_{1} \eta P_{B S}}{N_{0}} g_{1}^{2}}{1+\sum_{i=2}^{K} \frac{\Delta g_{i} \eta P_{B S}}{N_{0}} g_{i}^{2}}\right) \\
& =T \log _{2}\left(1+\frac{\Delta \rho g_{1}^{2}}{1+\sum_{i=2}^{K} \Delta \rho g_{i}^{2}}\right)
\end{aligned}
$$

Further it can be simplified as

$$
R_{1}=T \log _{2}\left(1+\frac{\Delta \rho g_{1}^{2}}{1+\Delta \rho \sum_{i=2}^{K} g_{i}^{2}}\right)
$$




\section{Bibliography}

[1] F. Boccardi, R. W. Heath, A. Lozano, T. L. Marzetta, and P. Popovski, "Five disruptive technology directions for 5G," Communications Magazine, IEEE, vol. 52, no. 2, pp. 74-80, 2014.

[2] A. Osseiran, F. Boccardi, V. Braun, K. Kusume, P. Marsch, M. Maternia, O. Queseth, M. Schellmann, H. Schotten, H. Taoka, et al., "Scenarios for 5G mobile and wireless communications: the vision of the METIS project," Communications Magazine, IEEE, vol. 52, no. 5, pp. 26-35, 2014.

[3] I. F. Akyildiz, S. Nie, S.-C. Lin, and M. Chandrasekaran, "5G roadmap: 10 key enabling technologies," Computer Networks, vol. 106, pp. 17-48, 2016.

[4] S. R. Islam, N. Avazov, O. A. Dobre, and K.-S. Kwak, "Power-domain non-orthogonal multiple access (noma) in 5g systems: Potentials and challenges," IEEE Communications Surveys \& Tutorials, 2016.

[5] Z. Ding, Y. Liu, J. Choi, Q. Sun, M. Elkashlan, H. V. Poor, et al., "Application of nonorthogonal multiple access in LTE and 5G networks," IEEE Communications Magazine, vol. 55, no. 2, pp. 185-191, Feb. 2017.

[6] A. Benjebbour, A. Li, K. Saito, Y. Saito, Y. Kishiyama, and T. Nakamura, "Noma: From concept to standardization," in Standards for Communications and Networking (CSCN), 2015 IEEE Conference on, pp. 18-23, IEEE, 2015. 
[7] L. Dai, B. Wang, Y. Yuan, S. Han, I. Chih-Lin, and Z. Wang, "Non-orthogonal multiple access for 5G: solutions, challenges, opportunities, and future research trends," Communications Magazine, IEEE, vol. 53, no. 9, pp. 74-81, Sep. 2015.

[8] K. Higuchi and A. Benjebbour, "Non-orthogonal multiple access (noma) with successive interference cancellation for future radio access," IEICE Transactions on Communications, vol. 98 , no. 3, pp. 403-414, 2015.

[9] P. Sedtheetorn and T. Chulajata, "Spectral efficiency evaluation for non-orthogonal multiple access in Rayleigh fading," in 2016 18th International Conference on Advanced Communication Technology (ICACT), pp. 747-750, IEEE, 2016.

[10] P. Sedtheetorn and T. Chulajata, "Spectral efficiency evaluation for non-orthogonal multiple access in Rayleigh fading," in 2016 18th International Conference on Advanced Communication Technology (ICACT), pp. 747-750, IEEE, 2016.

[11] Z. Yuan, G. Yu, W. Li, Y. Yuan, X. Wang, and J. Xu, "Multi-User Shared Access for Internet of Things," in 2016 IEEE 83rd Vehicular Technology Conference (VTC Spring), pp. 1-5, IEEE, 2016.

[12] S. Yang, P. Chen, L. Liang, Q. Bi, and F. Yang, "System design and performance evaluation for power domain non-orthogonal multiple access," in 2015 IEEE/CIC International Conference on Communications in China (ICCC), pp. 1-5, IEEE, 2015.

[13] Y. Saito, Y. Kishiyama, A. Benjebbour, T. Nakamura, A. Li, and K. Higuchi, "Nonorthogonal multiple access (NOMA) for cellular future radio access," in Vehicular Technology Conference (VTC Spring), 2013 IEEE 77th, pp. 1-5, IEEE, Jun. 2013.

[14] K. Higuchi and Y. Kishiyama, "Non-orthogonal access with random beamforming and intrabeam SIC for cellular MIMO downlink," in Vehicular Technology Conference (VTC Fall), 2013 IEEE 78th, pp. 1-5, IEEE, Sep. 2013. 
[15] S. Han, I. Chih-Lin, Z. Xu, and Q. Sun, "Energy efficiency and spectrum efficiency co-design: From NOMA to network NOMA,” E-LETTER, Sep. 2014.

[16] A. G. Perotti and B. M. Popovie, "Non-orthogonal multiple access for degraded broadcast channels: RA-CEMA," in Wireless Communications and Networking Conference (WCNC), 2015 IEEE, pp. 735-740, IEEE, Mar. 2015.

[17] H. Nikopour and H. Baligh, "Sparse code multiple access," in Personal Indoor and Mobile Radio Communications (PIMRC), 2013 IEEE 24th International Symposium on, pp. 332336, IEEE, Sep. 2013.

[18] R. Hoshyar, F. P. Wathan, and R. Tafazolli, "Novel low-density signature for synchronous CDMA systems over AWGN channel," Signal Processing, IEEE Transactions on, vol. 56, no. 4, pp. 1616-1626, Apr. 2008.

[19] M. Al-Imari, P. Xiao, M. A. Imran, and R. Tafazolli, "Uplink non-orthogonal multiple access for 5G wireless networks," in Wireless Communications Systems (ISWCS), 2014 11th International Symposium on, pp. 781-785, IEEE, Aug. 2014.

[20] J. Huang, K. Peng, C. Pan, F. Yang, and H. Jin, "Scalable video broadcasting using bit division multiplexing," Broadcasting, IEEE Transactions on, vol. 60, no. 4, pp. 701-706, Dec. 2014.

[21] Z. Ding, Z. Yang, P. Fan, and H. V. Poor, "On the performance of non-orthogonal multiple access in 5G systems with randomly deployed users," Signal Processing Letters, IEEE, vol. 21, no. 12, pp. 1501-1505, Jul. 2014.

[22] Y. Lan, A. Benjebboiu, X. Chen, A. Li, and H. Jiang, "Considerations on downlink nonorthogonal multiple access (NOMA) combined with closed-loop SU-MIMO," in Signal Processing and Communication Systems (ICSPCS), 2014 8th International Conference on, pp. 15, IEEE, Dec. 2014. 
[23] X. Chen, A. Benjebbour, Y. Lan, A. Li, and H. Jiang, "Impact of rank optimization on downlink non-orthogonal multiple access (NOMA) with SU-MIMO," in Communication Systems (ICCS), 2014 IEEE International Conference on, pp. 233-237, IEEE, Nov. 2014.

[24] S. Timotheou and I. Krikidis, "Fairness for non-orthogonal multiple access in 5G systems," Signal Processing Letters, IEEE, vol. 22, no. 10, pp. 1647-1651, Oct. 2015.

[25] T. Yunzheng, L. Long, L. Shang, and Z. Zhi, "A survey: Several technologies of nonorthogonal transmission for 5G," Communications, China, vol. 12, no. 10, pp. 1-15, Nov. 2015.

[26] B. Wang, K. Wang, Z. Lu, T. Xie, and J. Quan, "Comparison study of non-orthogonal multiple access schemes for 5g," in Broadband Multimedia Systems and Broadcasting (BMSB), 2015 IEEE International Symposium on, pp. 1-5, IEEE, 2015.

[27] W. Ejaz, M. Naeem, M. Basharat, A. Anpalagan, and S. Kandeepan, "Efficient wireless power transfer in software-defined wireless sensor networks," IEEE Sensors Journal, vol. 16, no. 20, pp. 7409-7420, Oct. 2016.

[28] W. Han, J. Ge, and J. Men, "Performance analysis for noma energy harvesting relaying networks with transmit antenna selection and maximal-ratio combining over nakagami-m fading," IET Communications, vol. 10, no. 18, pp. 2687-2693, 2016.

[29] A. A. Nasir, X. Zhou, S. Durrani, and R. A. Kennedy, "Relaying protocols for wireless energy harvesting and information processing," IEEE Transactions on Wireless Communications, vol. 12, no. 7, pp. 3622-3636, 2013.

[30] P. D. Diamantoulakis, K. N. Pappi, Z. Ding, and G. K. Karagiannidis, "Wireless-powered communications with non-orthogonal multiple access," IEEE Transactions on Wireless Communications, vol. 15, no. 12, pp. 8422-8436, Dec. 2016. 
[31] Y. Liu, Z. Ding, M. Elkashlan, and H. V. Poor, "Cooperative non-orthogonal multiple access with simultaneous wireless information and power transfer," IEEE Journal on Selected Areas in Communications, vol. 34, no. 4, pp. 938-953, 2016.

[32] S. Ali, E. Hossain, and D. I. Kim, "Non-orthogonal multiple access (noma) for downlink multiuser mimo systems: User clustering, beamforming, and power allocation,' IEEE Access, vol. 5, pp. 565-577, 2017.

[33] M. S. Ali, H. Tabassum, and E. Hossain, "Dynamic user clustering and power allocation for uplink and downlink non-orthogonal multiple access (noma) systems," IEEE Access, vol. 4, pp. 6325-6343, 2016.

[34] X. Zhang, Q. Gao, C. Gong, and Z. Xu, "User grouping and power allocation for noma visible light communication multi-cell networks," IEEE Communications Letters, vol. 21, no. 4, pp. 777-780, Apr. 2017.

[35] K. Wang, Z. Ding, and W. Liang, "A game theory approach for user grouping in hybrid non-orthogonal multiple access systems," in International Symposium on Wireless Communication Systems (ISWCS), pp. 643-647, IEEE, 2016.

[36] Z. Q. Al-Abbasi and D. K. So, "User-pairing based non-orthogonal multiple access (noma) system," in Vehicular Technology Conference (VTC Spring), pp. 1-5, IEEE, 2016.

[37] J. Kim, J. Koh, J. Kang, K. Lee, and J. Kang, "Design of user clustering and precoding for downlink non-orthogonal multiple access (noma)," in IEEE Military Communications Conference, MILCOM, pp. 1170-1175, IEEE, 2015.

[38] K. Yakou and K. Higuchi, "Downlink noma with sic using unified user grouping for nonorthogonal user multiplexing and decoding order," in Intelligent Signal Processing and Communication Systems (ISPACS), 2015 International Symposium on, pp. 508-513, IEEE, 2015. 
[39] Z. Yang, Z. Ding, P. Fan, and N. Al-Dhahir, “The Impact of Power Allocation on Cooperative Non-orthogonal Multiple Access Networks with SWIPT," IEEE Transactions on Wireless Communications, 2017.

[40] R. Sun, Y. Wang, X. Wang, and Y. Zhang, "Transceiver design for cooperative non-orthogonal multiple access systems with wireless energy transfer," IET Communications, vol. 10, no. 15, pp. 1947-1955, 2016.

[41] H. Chingoska, Z. Hadzi-Velkov, I. Nikoloska, and N. Zlatanov, "Resource allocation in wireless powered communication networks with non-orthogonal multiple access," IEEE Wireless Communications Letters, vol. 5, no. 6, pp. 684-687, 2016.

[42] P. D. Diamantoulakis, K. N. Pappi, Z. Ding, and G. K. Karagiannidis, “Optimal design of non-orthogonal multiple access with wireless power transfer," in Communications (ICC), 2016 IEEE International Conference on, pp. 1-6, IEEE, 2016.

[43] Y. Saito, A. Benjebbour, Y. Kishiyama, and T. Nakamura, "System-level performance evaluation of downlink non-orthogonal multiple access (noma)," in Personal Indoor and Mobile Radio Communications (PIMRC), 2013 IEEE 24th International Symposium on, pp. 611615, IEEE, 2013.

[44] H. Jin, K. Peng, and J. Song, “A Spectrum Efficient Multi-User Transmission Scheme for 5G Systems With Low Complexity," Communications Letters, IEEE, vol. 19, no. 4, pp. 613-616, Apr. 2015.

[45] Z. Ding, M. Peng, and H. V. Poor, "Cooperative non-orthogonal multiple access in 5G systems," Communications Letters, IEEE, vol. 19, no. 8, pp. 1462-1465, Aug. 2015.

[46] G. Song and X. Wang, "Comparison of Interference Cancellation Schemes for NonOrthogonal Multiple Access System,” in 2016 IEEE 83rd Vehicular Technology Conference (VTC Spring), pp. 1-5, IEEE, 2016. 
[47] D. W. K. Ng, E. S. Lo, and R. Schober, "Wireless information and power transfer: Energy efficiency optimization in OFDMA systems," IEEE Transactions on Wireless Communications, vol. 12, no. 12, pp. 6352-6370, Dec. 2013.

[48] S. G. Glisic and P. A. Leppänen, Wireless communications: TDMA versus CDMA. Springer Science \& Business Media, 2013.

[49] D. T. Ngo, L. B. Le, T. Le-Ngoc, E. Hossain, and D. I. Kim, "Distributed interference management in two-tier CDMA femtocell networks," IEEE Transactions on Wireless Communications, vol. 11, no. 3, pp. 979-989, Mar. 2012.

[50] P. Muneer and S. Sameer, "Iterative joint carrier frequency offset and doubly selective channel estimation in high-mobility mimo-ofdma uplink using oblique projection," IEEE Transactions on Vehicular Technology, vol. 65, no. 9, pp. 7110-7121, 2016.

[51] A. Mohamad, R. Visoz, and A. O. Berthet, "Outage achievable rate analysis for the non orthogonal multiple access multiple relay channel," in Wireless Communications and Networking Conference Workshops (WCNCW), 2013 IEEE, pp. 160-165, IEEE, Apr. 2013.

[52] N. Bhuvanasundaram, H. X. Nguyen, R. Trestian, and Q.-T. Vien, "Sum-rate analysis of cell edge users under cooperative NOMA," in 2015 8th IFIP Wireless and Mobile Networking Conference (WMNC), pp. 239-244, IEEE, Oct. 2015.

[53] Y. Xu, H. Sun, R. Q. Hu, and Y. Qian, "Cooperative Non-Orthogonal Multiple Access in Heterogeneous Networks," in 2015 IEEE Global Communications Conference (GLOBECOM), pp. 1-6, IEEE, Dec. 2015.

[54] J.-B. Kim and I.-H. Lee, "Non-Orthogonal Multiple Access in Coordinated Direct and Relay Transmission," IEEE Communications Letters, vol. 19, no. 11, pp. 2037-2040, Nov. 2015. 
[55] C. Wang, M. Xiao, and M. Skoglund, "Diversity-multiplexing tradeoff analysis of multisource multi-relay coded networks," in Information Theory and its Applications (ISITA), 2010 International Symposium on, pp. 422-427, IEEE, Oct. 2010.

[56] S. Wei, J. Li, W. Chen, H. Su, Z. Lin, and B. Vucetic, "Power adaptive network coding for a non-orthogonal multiple-access relay channel," Communications, IEEE Transactions on, vol. 62, no. 3, pp. 872-887, Mar. 2014.

[57] S. Wei, J. Li, and W. Chen, "Network coded power adaptation scheme in non-orthogonal multiple-access relay channels," in Communications (ICC), 2014 IEEE International Conference on, pp. 4820-4825, IEEE, Jun. 2014.

[58] S. Wei, J. Li, W. Chen, L. Zheng, and H. Su, "Design of generalized analog network coding for a multiple-access relay channel," Communications, IEEE Transactions on, vol. 63, no. 1, pp. 170-185, Dec. 2015.

[59] J. Men, J. Ge, and C. Zhang, "Performance Analysis of Non-Orthogonal Multiple Access for Relaying Networks Over Nakagami-m Fading Channels," Apr. 2015.

[60] J.-B. Kim and I.-H. Lee, "Capacity Analysis of Cooperative Relaying Systems Using NonOrthogonal Multiple Access," IEEE Communications Letters, vol. 19, no. 11, pp. 1949-1952, Nov. 2015.

[61] A. Mohamad, R. Visoz, and A. O. Berthet, "Code design for multiple-access multiple-relay wireless channels with non-orthogonal transmission," in 2015 IEEE International Conference on Communications (ICC), pp. 2318-2324, IEEE, Jun. 2015.

[62] A. Hatefi, R. Visoz, and A. O. Berthet, "Near outage limit joint network coding and decoding for the Non-Orthogonal Multiple-Access Relay Channel," in Personal Indoor and Mobile Radio Communications (PIMRC), 2012 IEEE 23rd International Symposium on, pp. 18671873, IEEE, Sep. 2012. 
[63] H. Kwon, J. Lee, and I. Kang, "Successive interference cancellation via rank-reduced maximum a posteriori detection," Communications, IEEE Transactions on, vol. 61, no. 2, pp. 628637, 2013.

[64] L.-Y. Song and A. G. Burr, "Successive interference cancelation for space-time block codes over time-selective channels," IEEE communications letters, vol. 10, no. 12, pp. 837-839, 2006.

[65] I. S. Park and S.-Y. Chung, "On the Diversity-Multiplexing Tradeoff Under Groupwise Successive Interference Cancelation," Communications, IEEE Transactions on, vol. 59, no. 10, pp. 2862-2870, 2011.

[66] G. D. Forney and L.-F. Wei, "Multidimensional constellations. I. Introduction, figures of merit, and generalized cross constellations," IEEE journal on selected areas in communications, vol. 7, no. 6, pp. 877-892, 1989.

[67] G. D. Forney, "Multidimensional constellations. II. Voronoi constellations," IEEE Journal on Selected Areas in Communications, vol. 7, no. 6, pp. 941-958, 1989.

[68] Z. Ding, R. Schober, and H. V. Poor, “A General MIMO Framework for NOMA Downlink and Uplink Transmission Based on Signal Alignment," arXiv preprint arXiv:1508.07433, Aug, 2015.

[69] A.-I. Mohammed, M. Imran, and P. Xiao, "Radio Resource Allocation for Multicarrier-Low Density Spreading Multiple Access,” Jun. 2016.

[70] V. Kalokidou, O. Johnson, and R. Piechocki, "A hybrid TIM-NOMA scheme for the SISO Broadcast Channel," in Communication Workshop (ICCW), 2015 IEEE International Conference on, pp. 387-392, IEEE, Jun. 2015. 
[71] L. Yao, J. Mei, H. Long, L. Zhao, and K. Zheng, "A novel multi-user grouping scheme for downlink non-orthogonal multiple access systems," in IEEE Wireless Communications and Networking Conference (WCNC), pp. 1-6, IEEE, 2016.

[72] M. F. Kader, M. B. Shahab, and S. Y. Shin, "Cooperative spectrum sharing with energy harvesting best secondary user selection and non-orthogonal multiple access," in Computing, Networking and Communications (ICNC), 2017 International Conference on, pp. 46-51, IEEE, 2017.

[73] Y. Liu, Z. Ding, M. Eikashlan, and H. V. Poor, "Cooperative non-orthogonal multiple access in 5g systems with swipt," in Signal Processing Conference (EUSIPCO), 2015 23rd European, pp. 1999-2003, IEEE, Dec. 2015.

[74] J. Konecnỳ and P. Richtárik, “Simple complexity analysis of direct search,” CoRR, 2014.

[75] M. A. Abramson, C. Audet, J. W. Chrissis, and J. G. Walston, "Mesh adaptive direct search algorithms for mixed variable optimization," Optimization Letters, vol. 3, no. 1, pp. 35-47, 2009.

[76] C. Yan, A. Harada, A. Benjebbour, Y. Lan, A. Li, and H. Jiang, "Receiver Design for Downlink Non-Orthogonal Multiple Access (NOMA)," in Vehicular Technology Conference (VTC Spring), 2015 IEEE 81st, pp. 1-6, IEEE, May. 2015.

[77] M. Xu, F. Ji, M. Wen, and W. Duan, "Novel Receiver Design for the Cooperative Relaying System with Non-Orthogonal Multiple Access," IEEE Communications Letters, vol. 20, no. 8 , pp. 1679-1682.

[78] Z. Yang, Z. Ding, P. Fan, and G. K. Karagiannidis, “On the Performance of Non-orthogonal Multiple Access Systems With Partial Channel Information,” IEEE Transactions on Communications, vol. 64, no. 2, pp. 654-667, 2016. 
[79] W. Xi and H. Zhou, "Enhanced CSI feedback scheme for non-orthogonal multiple access," in 2016 Wireless Days (WD), pp. 1-3, IEEE, 2016.

[80] Z. Ding, L. Dai, and H. V. Poor, "MIMO-NOMA design for small packet transmission in the Internet of things," IEEE Access, vol. 4, pp. 1393-1405, 2016.

[81] Z. Ding, P. Fan, and V. Poor, "Impact of User Pairing on 5G Non-Orthogonal Multiple Access Downlink Transmissions," IEEE Transactions on Vehicular Technology, vol. 65, no. 8, pp. 6010-6023, Aug. 2016.

[82] P. D. Diamantoulakis, K. N. Pappi, Z. Ding, and G. K. Karagiannidis, "Optimal design of nonorthogonal multiple access with wireless power transfer," arXiv preprint arXiv:1511.01291, 2015.

[83] Y. Yuan and Z. Ding, "The application of non-orthogonal multiple access in wireless powered communication networks," in 2016 IEEE 17th International Workshop on Signal Processing Advances in Wireless Communications (SPAWC), pp. 1-5, IEEE, 2016. 